\title{
Punctures and $p$-Spin Curves from Matrix Models
}

\author{
E. Brézin ${ }^{1} \cdot$ S. Hikami ${ }^{2}$
}

Received: 27 January 2020 / Accepted: 28 May 2020 / Published online: 6 June 2020

(c) The Author(s) 2020

\begin{abstract}
This article investigates the intersection numbers of the moduli space of $p$-spin curves with the help of matrix models. The explicit integral representations that are derived for the generating functions of these intersection numbers exhibit $p$ Stokes domains, labelled by a "spin"-component $l$ taking values $l=-1,0,1,2, \ldots, p-2$. Earlier studies concerned integer values of $p$, but the present formalism allows one to extend our study to half-integer or negative values of $p$, which turn out to describe new types of punctures or marked points on the Riemann surface. They fall into two classes: Ramond $(l=-1)$, absent for positive integer $p$, and Neveu-Schwarz $(l \neq-1)$. The intersection numbers of both types are computed from the integral representation of the $n$-point correlation functions in a large $N$ scaling limit. We also consider a supersymmetric extension of the random matrix formalism to show that it leads naturally to an additional logarithmic potential. Open boundaries on the surface, or admixtures of R and NS punctures, may be handled by this extension.
\end{abstract}

Keywords Random matrices · Matrix models, Intersection Numbers, Large N

\section{Introduction}

Although the connection between matrix models and the geometry of surfaces is by now an old subject, originating with the work of 't Hooft [1] for large $\mathrm{N}$ gauge theories, new features

Communicated by Ivan Corwin.

To our very dear and highly esteemed friend Joel Lebowitz. "Se questo è un uomo" asked Primo Levi in 1947. No one is more an "uomo" than Joel.

$凶$ E. Brézin

brezin@1pt.ens.fr

S. Hikami

hikami@oist.jp

1 Laboratoire de Physique de l'Ecole normale supérieure, ENS, Université PSL, CNRS, Sorbonne Université, Université de Paris, 75005 Paris, France

2 Okinawa Institute of Science and Technology Graduate University, 1919-1 Tancha, Okinawa 904-0495, Japan 
have been revealed recently involving super-Riemann surfaces with punctures or boundaries, related to matrix models with various types of symmetries [2].

In this work we return to the use of Gaussian matrix models with an external matrix source which, according to the nature of the source, covers a large spectrum of applications. For instance we have considered earlier an external source which generates a gapped density of eigenvalues, and a critical point at the closing gap situation [3]. In the course of this work this closing gap situation corresponds to the $p=3$ model defined below. As a quantum topological field theory, we have shown earlier that it provides a generating function of the intersection numbers in the moduli space of Riemann surface of $p$-spin curves [4-8]. This $p$ spin curve is described by an Hermitian matrix model involving the $(p+1)$-th power of matrix, with an external source tuned appropriately [8], in a large matrix-size scaling limit. The model is thus a generalization of the Kontsevich Airy matrix model $(p=2)$ [9]. In this work we have not attempted to give proofs relating the geometry of Riemann surfaces to the generalized matrix integrals considered below. Our contribution consists simply of making use of previous results concerning matrix models in an external matrix source appropriately tuned to provide numerical values for intersection numbers in various new situations.

In this article, we consider the extension to half-integer spins such, $p=\frac{1}{2}, \frac{3}{2}, \ldots$, and to negative integers for which new features appear [8,10,11]. Positive integer $p$ corresponds to a compact coset space $S U(2) / U(1)$ with level $k=p-2$ of the WZW model, and negative $p$ to the non-compact, hyperbolic space, $S L(2, R) / U(1)$, as explained by Witten $[12,13]$.

When $p=-1$, the intersection numbers reduce to the Euler characteristics of orbifolds $[6,14,15]$, and when $p=-2$ the model is equivalent to a unitary matrix model with a $U(N)$ gauge field [8,10,16-20]. (A simple proof of the equivalence between a unitary matrix model with the $p=-2$ model may be found in the Appendix $C$ of [21], where it was shown that they share the same equations of motion).

The case of half-integer $p$, obtained by continuation from integer $p$, provides new features. The fractional level case corresponds to a fractional level $k$ of the WZW model, which is a nonunitary conformal field theory [22-24]. It is also related to the super ghost $\beta-\gamma$ system, within a superconformal field theory [25]. Another supersymmetric derivation of the intersection numbers of $p$-spin curves, which is different from the present article, may be found in [2628]. The case half-integer $p$ is also interesting as it realizes a Chern-Simons theory coupled to a Majorana fermion. It has been discussed earlier that the presence of fermions leads to the shift of the level $k \rightarrow k-\frac{1}{2}$, see for instance [29]. The spin $p=\frac{1}{2}$ case may thus be interpreted as $p=1-\frac{1}{2}$, where the first term $p=1$ comes from a simple Gaussian case. Similarly $p=-\frac{1}{2}$ may be interpreted as a pure fermion contribution, i.e. $p=0-\frac{1}{2}$. For both cases, we show that the intersection numbers involve Ramond punctures. There are many contributions related to the geometry of Riemann surfaces, see among them [30,31].

Ramond (R) and Neveu-Schwarz (NS) punctures come from the study of string theory over super-Riemann surfaces [32,33]. Two types of nodal points can be defined on a Riemann surface. If a line bundle at such a point is locally free (i.e. there is no orbifold structure or in our approach no Stokes lines) it is called R-type; otherwise (non-trivial orbifold structure) it belongs to the NS- type. In this article, we use these terminologies for the two distinct types of punctures (or marked points). The contour integrals which describe the intersection numbers with these punctures turn out to be different. For the NS- type, the component $l$ of spin $p$ takes one of the values $(0,1, \ldots, p-2)$, and the R-type $l$ corresponds to the single value $l=-1$ [34,35], which distinguishes the orbifold structures. We consider $s$ punctures on a Riemann surface, and from a dimensional counting, a selection rule (Riemann-Roch, denoted RR) relates th genus $g$, the spin $p$ and the indices $n_{i}, l_{i}(i=1, \ldots, s)$. It is well 
known that there are no contributions of R-type for positive integer $p[7,36]$ as it will be shown in Eq. (19) of Sect. 2.

We have also found an R-contribution in the presence of an additional logarithmic potential [7,21]. The R-type is related to the coefficients $m$ of the logarithmic potential of the matrix model, in which the power $m^{b}$, represents the number of open boundaries of the Riemann surface. One aim of this article is to discuss more systematically the appearance of R-type punctures on the basis of the results of [7,21], in which open intersection numbers have already been considered.

The calculations of intersection numbers of R-type reduce to computing residues from an integral representation. When $p \in Z+\frac{1}{2}$ (half-integer), the moduli space becomes a spin moduli space, and it is related for the lowest fractions to a Dirac spin $\left(p=\frac{1}{2}\right)$ or a Rarita-Schwinger operator $\left(p=\frac{3}{2}\right)[37,38]$.

When $p=-1 / 2$, all intersection numbers with one marked point vanish for a genus $g>1$, since all the intersection numbers of $p$-spin curves involve a $(2 p+1)$-factor. This behavior was noticed earlier from explicit expressions previously obtained up to genus 9 [8]. We prove here that for $p=-1 / 2$ the intersection numbers vanish to all orders in the genus $(g>1)$.

We have also considered the formalism of random supermatrices in an external source introduced in a previous article [39]. There the tuning of the source in a scaling limit generates a matrix model with and additional logarithmic potential term, a generalized KontsevichPenner model. The open intersection numbers had been computed earlier [21]. In this case, the R-type $(l=-1)$ does appear in the intersection numbers $\tau_{n,-1}$. (In [21], it was denoted as $\tau_{n-\frac{1}{2}}$ ). These R-type punctures are related to the odd or even character of the power of $m$, in the coefficient of the logarithmic potential. For $p= \pm \frac{1}{2}$, an R-puncture is interpreted as a fermion on the boundaries; an odd number of R-punctures appears with an odd number of boundaries, and an even number of R-punctures only with even powers of $m$. Even powers $b$ for $m^{b}$ correspond to an even number of boundaries.

We also discuss a supersymmetric model with admixtures of positive and negative powers $p$ for the matrices, in addition to a logarithmic term. The negative integer $p$, and half-integer $p$, matrix models were recently proposed in the context of irregular conformal blocks $[40,41]$ : we briefly mention this correspondence in Sect. 5. The super-matrix formulation may shed light on the study of the extension of $A_{n}$ singularities to other ADE cases [36], but such investigations are left for a future study.

This article consists of the following sections. In Sect. 2, we briefly review the formulae for the $n$-point functions in presence of an external source, and how its tuning can generate a $p$-spin curve and provide the intersection numbers. In Sect. 3, we consider the one-point function. The extension to half-integer $p$ yields punctures of R-type. In Sect. 4, we study the two point correlation function for positive integer spin $p$ and half-integer $p=\frac{1}{2}$ and obtain the intersection numbers. In Sect. 5, we discuss super-matrix models. Within such models, the two point correlation function is computed for $p=\frac{1}{2}$ (Ramond) and $p=2$ (Neveu-Schwarz). In Sect. 6, open intersection numbers are discussed through a logarithmic potential. Section 7 is devoted to a summary and discussions. In an Appendix, we consider the intersection numbers for logarithmic potentials from Virasoro equations. 


\section{Intersection Numbers for $p$-Spin Curves}

The $p$-spin matrix model is a generalization of Kontsevich Airy matrix model [9] defined by

$$
Z=\int d B e^{-\frac{1}{p+1} \operatorname{Tr} B^{p+1}+\operatorname{Tr} B \Lambda}
$$

where $B$ is a $k \times k$ Hermitian matrix, and $\Lambda$ a fixed $k \times k$ Hermitian matrix. The matrix integral is well defined only for odd $p$. However we consider here those matrix integrals for general $p$ as generating functions, a bookkeeping of expectation values as for instance in the loop equations. Furthermore exact solutions of matrix models with polynomial potentials show that, in the limit of matrices of infinite size, the result remains analytic in domains of instability of the potential [42]. For the $p=2$ model Kontsevich dealt with an $i B^{3}$ theory. We could have done the same here, but this would not change the algebraic relations discussed below. Furthermore this would not save us for fractional $p$.

For the $p=2$ model, Kontsevich proved that $\log Z$, expanded in products of inverse powers of the $\operatorname{Tr}\left(1 / \Lambda^{n}\right)$, regarded as independent for large matrices, was a generating function of the intersection numbers of the moduli of curves on a Riemann surface. The aim of this article is to derive those expansions for arbitrary $p$, a priori a positive integer, but continued also to fractional or negative values. The method consists of using two exact results a) an $N-k$ duality between $k$ point functions of characteristic polynomials for $N \times N$ Hermitian matrices in an external matrix source b) explicit formulae for $k$ point functions for a Gaussian model in an external matrix source. These two results are summarized below.

We consider in the following the average of a product of characteristic polynomials of the Hermitian $N \times N$ matrix $M$ with an external source $A$ defined by

$$
\begin{aligned}
F_{k}\left(\lambda_{1}, \ldots, \lambda_{k}\right) & =\frac{1}{Z_{N}}\left\langle\prod_{\alpha=1}^{k} \operatorname{det}\left(\lambda_{\alpha} \cdot I-M\right)\right\rangle_{A, M} \\
& =\frac{1}{Z_{N}} \int d M \prod_{\alpha=1}^{k} \operatorname{det}\left(\lambda_{\alpha} \cdot I-M\right) e^{-\frac{N}{2} \operatorname{tr} M^{2}+N \operatorname{tr} M A}
\end{aligned}
$$

where one averages over the $N \times N$ matrices $M ; A$ is a given Hermitian matrix, whose eigenvalues are $\left(a_{1}, \ldots, a_{N}\right) . I$ is the identity matrix and $Z_{N}$ is the normalization constant of the probability measure for $A=0$. The duality formula derived in $[4,43]$ gives another matrix integral for the same average

$$
F_{k}\left(\lambda_{1}, \ldots, \lambda_{k}\right)=\frac{1}{Z_{k}} \int d B \prod_{j=1}^{N} \operatorname{det}\left(a_{j}-i B\right) e^{-\frac{N}{2} \operatorname{Tr}(B-i \Lambda)^{2}}
$$

where $B$ is a $k \times k$ Hermitian matrix and $\Lambda=\operatorname{diag}\left(\lambda_{1}, \ldots, \lambda_{k}\right)$ a source matrix; (we use the notation ' $T r$ ' for traces of $k \times k$ matrices and 'tr' for those $N \times N)$. The normalization constant is $Z_{k}=\int d B \exp \left(-\frac{N}{2} \operatorname{Tr} B^{2}\right)$. The proof is given in the Appendix B of [4].

Let us now show that the freedom provided by the $N$ eigenvalues of the source $A$ may be used to tune the dual $B$ model (3) to the p-spin matrix model (1). For that purpose it is sufficient to specialize those formulae to an external matrix source A possessing $(p-1)$ distinct eigenvalues $A=\left(a_{1}, . ., a_{1}, \ldots, a_{p-1}, \ldots ., a_{p-1}\right)$. For simplicity we have assumed that every distinct eigenvalue is degenerate $N /(p-1)$ times. Furthermore we now fix the 
eigenvalues of $\mathrm{A}$ by the conditions

$$
\begin{aligned}
& \sum_{i=1}^{p-1} \frac{1}{a_{i}^{2}}=p-1, \quad \sum_{i=1}^{p-1} \frac{1}{a_{i}^{m}}=0 \quad(m=3,4, \ldots, p) \\
& \sum_{i=1}^{p-1} \frac{1}{a_{i}^{p+1}} \neq 0 .
\end{aligned}
$$

For example consider the case $p=3$. Choosing $a_{1}=+1, a_{2}=-1$, one has $1 / a_{1}^{2}+1 / a_{2}^{2}=2$, $1 / a_{1}^{3}+1 / a_{2}^{3}=0,1 / a_{1}^{4}+1 / a_{2}^{4} \neq 0$ which satisfy the conditions (4).

Dealing with the B-side of the duality (3) we consider the expansion of $\prod_{j=1}^{N} \operatorname{det}\left(a_{j}-i B\right)$ in inverse powers of the $a_{j}$ :

$$
\prod_{j=1}^{N} \operatorname{det}\left(1-i B / a_{j}\right)=e^{-\frac{N}{p-1} \sum_{l=1}^{\infty} \frac{1}{l} \operatorname{Tr}(i B)^{l} \sum_{j=1}^{p-1} 1 / a_{j}^{l}}
$$

For definiteness consider the $p=3$ case; this expansion yields

$$
\prod_{j=1}^{N} \operatorname{det}\left(1-i B / a_{j}\right)=e^{N \operatorname{Tr}\left(\frac{1}{2} B^{2}-\frac{1}{4} B^{4}+\cdots\right)}
$$

The $B^{2}$ terms in the exponential of (3) cancels with the one in (5) and one is left with $e^{N \operatorname{Tr}\left(i \Lambda B-\frac{1}{4} B^{4}+\cdots\right)}$. This is precisely the critical gap closing model studied in detail in [3]. Following Kontsevich [9] we want to expand the $B$-integral (more precisely the free energy $\log Z$ ) in powers of $\operatorname{Tr}\left(1 / \Lambda^{n}\right)$. To this effect we take a scaling limit in which the eigenvalues of $\Lambda$ are of order $N^{-3 / 4}$ and the matrix elements of $B$ of order $N^{-1 / 4}$ in the large $N$ limit. In this scaling limit all the higher terms of the form $N \operatorname{Tr} B^{m}, m>4$, in (5) are negligible.

For general $p$, following the conditions (4), the scaling limit is given by $\Lambda \sim N^{-\frac{p}{p+1}}$ and $B \sim N^{-\frac{1}{p+1}}$ and the expansion of (5) stops with the highest term $N \operatorname{Tr} B^{p+1}$. The two remaining terms $N \operatorname{Tr} \Lambda B$ and $N \operatorname{Tr} B^{p+1}$ are both of order one. So in the scaling limit the $B$-side of the duality $(2,3)$ is indeed a generalized Kontsevich model (1).

It remains to compute the intersection numbers from the expansion of $F_{k}\left(\lambda_{1}, \ldots, \lambda_{k}\right)$ in powers of the

$$
\bar{t}_{n}=\operatorname{Tr} \frac{1}{\Lambda^{n}} .
$$

i.e. as the coefficients of $\operatorname{Tr} \frac{1}{\Lambda^{n_{1}}} \operatorname{Tr} \frac{1}{\Lambda^{n_{2}}} \cdots$.

Now we appeal to the duality to compute them from the $M$-side of the duality(2). If we expand $\prod_{\alpha=1}^{k} \operatorname{det}\left(\lambda_{\alpha} \cdot I-M\right)$ in inverse powers of the $\lambda_{i}$ 's, one can reconstruct these coefficients by combinatorics of the expectation values $\left\langle\operatorname{tr} M^{q_{1}} \operatorname{tr} M^{q_{2}} \cdots\right\rangle$, given the relation

$$
\left\langle\prod_{a=1}^{k} \operatorname{det}\left(1-\frac{M}{\lambda_{a}}\right)\right\rangle=\left\langle e^{\sum_{a=1}^{k} \operatorname{tr} \log \left(1-M / \lambda_{a}\right)}\right\rangle=\left\langle e^{-\sum_{n=1}^{\infty} \frac{\bar{t}_{n} \mathrm{tr} M^{n}}{n}}\right\rangle
$$

where the bracket stands for an expectation value with the weight (2). Therefore if the logarithm of the partition function (1) is expanded in terms of the $\bar{t}_{n_{1}} \bar{t}_{n_{2}} \cdots$, the coefficients, i.e. the intersection numbers, are given by the connected expectation values $\left\langle\operatorname{tr} M^{n_{1}} \operatorname{tr} M^{n_{2}} \cdots\right\rangle_{c}$. 
If the expression of $\left\langle\operatorname{tr} M^{n_{1}} \cdots \operatorname{tr} M^{n_{q}}\right\rangle$ as functions of the $a_{j}$ 's is not simple, we know from our previous work an exact expression for the n-point correlation functions

$$
U\left(\sigma_{1}, \ldots, \sigma_{n}\right)=\left\langle\operatorname{tr} e^{\sigma_{1} M} \cdots \operatorname{tr} e^{\sigma_{n} M}\right\rangle
$$

as function of the eigenvalues $a_{j}$. These correlation functions are generating functions of the expectation values $\left\langle\operatorname{tr} M^{n_{1}} \cdots \operatorname{tr} M^{n_{q}}\right\rangle$. One can then reconstruct the expansion of $F_{k}$ in powers of the $1 / \lambda_{i}$ 's from the expansions of the $U$ 's in powers of the $\sigma$ 's.

Indeed for arbitrary eigenvalues $a_{j}$ of the source matrix $A$, we have found in [5] that

$$
U(\sigma)=\left\langle\operatorname{tr} e^{\sigma M}\right\rangle=\frac{1}{N \sigma} e^{\frac{N}{2} \sigma^{2}} \oint \frac{d u}{2 i \pi} \prod_{j=1}^{N}\left(1-\frac{\sigma}{a_{j}-u}\right) e^{N \sigma u}
$$

where the contour in the u-plane encloses the eigenvalues $a_{j}$. Similarly the n-point correlation function $U\left(\sigma_{1}, \ldots, \sigma_{n}\right)$ are given by contour integrals over n complex variables $\sigma_{i}$,

$$
\begin{aligned}
& U\left(\sigma_{1}, \ldots, \sigma_{n}\right)=\left\langle\operatorname{tr} e^{\sigma_{1} M} \cdots \operatorname{tr} e^{\sigma_{n} M}\right\rangle \\
& =e^{\sum_{1}^{n} \sigma_{i}^{2}} \oint \prod_{i=1}^{n} \frac{d u_{i}}{2 i \pi} e^{\sum_{1}^{n} u_{i} \sigma_{i}} \prod_{\alpha=1}^{N} \prod_{i=1}^{n}\left(1-\frac{\sigma_{i}}{a_{\alpha}-u_{i}}\right) \operatorname{det} \frac{1}{u_{i}-u_{j}+\sigma_{i}}
\end{aligned}
$$

The contours are taken around the poles $u_{i}=a_{\alpha}$, not around the poles which come from the determinant.

We now specialize those formulae to the above external matrix source A possessing $(p-1)$ distinct eigenvalues $A=\left(a_{1}, . ., a_{1}, \ldots, a_{p-1}, \ldots ., a_{p-1}\right)$ satisfying the constraints (4). Then we expand, for reasons specified below,

$$
\prod_{j=1}^{N}\left(1-\frac{\sigma}{a_{j}-u}\right)=e^{\frac{N}{p-1} \sum_{1}^{p-1} \log \left(1-\frac{\sigma}{a_{j}-u}\right)}
$$

in inverse powers of the $1 / a_{j}$, taking $\sigma_{j}$ and $u$ as small and same order. For instance if $p=3$, with the conditions (4), the term $e^{N \sigma u}$ in (9) cancels and, the leading term in the exponent is $e^{N / 4\left[u^{4}-(u+\sigma)^{4}\right]}$. In the large $N$ scaling range in which $N \sigma \Lambda \sim N^{0}$, i.e. $\sigma \sim N^{-1 / 4}$, we can neglect the powers in $u$ and $\sigma$ higher than 4 .

For arbitrary $p$, with this choice of the matrix source A, in the scaling range under consideration $\Lambda \sim N^{-\frac{p}{p+1}}, \sigma$ and $u$ of order $N^{-1 /(p+1)}$, we obtain a generating function for the one-point intersection numbers of $p$-spin curves (i.e. the coefficients of single trace operators $\operatorname{Tr}\left(1 / \Lambda^{n}\right)$ from the integral (3),

$$
U(\sigma)=\frac{1}{N \sigma} \exp \left[-\frac{N}{p-1} \sigma\left(\sum_{j=1}^{p-1} \frac{1}{a_{j}}\right)\right] \int \frac{d u}{2 i \pi} e^{C\left[u^{p+1}-(u+\sigma)^{p+1}\right]}
$$

with $C=\frac{N}{p^{2}-1} \sum_{i=1}^{p-1} \frac{1}{a_{i}^{p+1}}$. If we choose further the condition $\sum \frac{1}{a_{j}}=0$, the exponential factor in the front of the integral can be omitted. The asymptotic behaviour under consideration depends of the integration path in the u-plane. Different paths correspond to different asymptotic limits and we need to consider them all. So at this stage we leave the integration path unspecified and return to it later.

With the conditions (4), the average of the characteristic polynomials for the matrix $B$ of (3) turns into the $p$-th generalized Kontsevich model (1). In this matrix model (1), we consider the eigenvalues $\lambda_{j}$ of the source matrix $\Lambda$ as large, and the intersection numbers are 
obtained from a $1 / \Lambda$ expansion. In the dual formulation the large $\Lambda$ expansion corresponds to an expansion of $U(\sigma)$ for small $\sigma$.

In the large $N$ limit we obtain, with this choice of the matrix source $\mathrm{A}$, a generating function for the intersection numbers of $p$-spin curves [5],

$$
U(\sigma)=\frac{1}{\sigma} \int \frac{d u}{2 i \pi} e^{C\left[u^{p+1}-(u+\sigma)^{p+1}\right]}
$$

or, after translation,

$$
U(\sigma)=\frac{1}{\sigma} \int \frac{d u}{2 i \pi} e^{C\left[(u-\sigma / 2)^{p+1}-(u+\sigma / 2)^{p+1}\right]}
$$

with $C=\frac{N}{p^{2}-1} \sum_{i=1}^{p-1} \frac{1}{a_{i}^{p+1}}$. Since we have expanded the integrand of (9) in the scaling limit $u \sim \sigma \sim N^{-1 /(p+1)}$ the contours for the re-scaled $u_{i}$ 's are now taken along specific paths needed to give a meaning to those expressions. The choice of the integration paths in the $\mathrm{u}$-plane will determine the nature of the asymptotics. This will be exemplified by the $p=3$ case which leads to Airy functions. For this $p$-spin model, the asymptotic expansion in $\sigma$ is governed by which of $p$ Stokes regions one is considering in the complex $u$-plane. The asymptotic behavior of the integrand in (13) being an exponential of $\sigma u^{p}$ there are $p$ sectors labeled by an index from 0 to (p-1) or, in accordance with the mathematical literature, a "spin"-index $l=-1,0, \ldots, p-2,[34,35]$.

Let us first consider the case $p=3$ for which (13) leads to an Airy function $A_{i}(\zeta$ ) (as in [4])

$$
U(\sigma)=\frac{1}{(N \sigma)^{4 / 3} 3^{1 / 3}} \mathrm{~A}_{\mathrm{i}}(\zeta)
$$

with $\zeta=-N^{2 / 3} 4^{-1} 3^{-1 / 3} \sigma^{8 / 3}$. The Airy function $A_{i}(x)$ is defined by

$$
A_{i}\left((3 a)^{-\frac{1}{3}} x\right)=\frac{(3 a)^{1 / 3}}{\pi} \int_{0}^{\infty} d t \cos \left(a t^{3}+x t\right)
$$

The standard expansion of the Airy function for small $x$ consists of two distinct series,

$$
\begin{aligned}
U(\sigma)= & \frac{1}{(N \sigma)^{4 / 3} 3^{1 / 3}}\left[\mathrm{~A}_{\mathrm{i}}(0)\left(1+\frac{1}{3 !} \zeta^{3}+\frac{1 \cdot 4}{6 !} \zeta^{6}+\frac{1 \cdot 4 \cdot 7}{9 !} \zeta^{9}+\cdots\right)\right. \\
& \left.+\mathrm{A}^{\prime}{ }^{\prime}(0)\left(\zeta+\frac{2}{4 !} \zeta^{4}+\frac{2 \cdot 5}{7 !} \zeta^{7}+\frac{2 \cdot 5 \cdot 8}{10 !} \zeta^{10}+\cdots\right)\right]
\end{aligned}
$$

where the first series corresponds to the spin-index $l=1$ with $\sigma^{m+\frac{2}{3}}$, and the second series to a spin-index $l=0$ with $\sigma^{m+\frac{1}{3}}$. The overall factors are $A i(0)=\frac{1}{2 \pi 3^{1 / 3}} \Gamma\left(\frac{1}{3}\right)$ and $A i^{\prime}(0)=$ $-\frac{1}{2 \pi} \Gamma\left(\frac{2}{3}\right)$.

A priori a third series with spin-index $l=-1$ could have been present, which would correspond to an asymptotic expansion of the form $\zeta^{2+3 m}(\mathrm{~m}=0,1,2, \ldots)$, leading to $\sigma^{\frac{8}{3}(2+3 m)-\frac{4}{3}}=$ $\sigma^{4+8 m}$, but such "Ramond" contribution is absent from the final asymptotics. 
Similarly for arbitrary $p$, there are terms labeled by the spin index $l$. The asymptotic expansion is generated by the integral

$$
\begin{aligned}
U(\sigma)= & \frac{1}{N \sigma} \int \frac{d u}{2 i \pi} \exp \left[-c \sigma u^{p}\right] \\
& \times \exp \left[-c\left(\frac{p(p-1)}{3 ! 4} \sigma^{3} u^{p-2}+\frac{p(p-1)(p-2)(p-3)}{5 ! 4^{2}} \sigma^{5} u^{p-4}+\cdots\right)\right]
\end{aligned}
$$

where the constant $c$ is related to $C$ in $(13): c=(p+1) C$, namely

$$
c=\frac{N}{p-1} \sum_{i=1}^{p-1} \frac{1}{a_{i}^{p+1}}
$$

a number proportional to N. Expanding in powers of $\sigma$ the second exponential and changing the integration variable $u \rightarrow(c \sigma)^{-1 / p} u^{1 / p}$, with a phase which depends of the Stokes sector under consideration, one obtains for the $l$-th sector

$$
U_{l}(\sigma)=\frac{1}{N} \sum_{n}\left\langle\tau_{n, l}\right\rangle \frac{1}{n \pi} \Gamma\left(1-\frac{1}{p}-\frac{l}{p}\right) c^{\frac{n+(l+1) / p}{p+1}} p^{1+\frac{p n+l+1}{p+1}} \sigma^{n+(l+1) / p}
$$

in which the spin index $l$ is a fixed number, taking one of the values -1 to $p-2$. The Gamma function in (19) and the power of $p$ have been extracted to match the conventional normalization of the intersection numbers $\left\langle\tau_{n, l}\right\rangle$.

The result is presented in (19) after expansion of the second exponential in (17). However it is interesting to return to the meaning of this expansion in terms of the $1 / N$ expansion. The coefficient $c$ is proportional to $N$ and the expansion is performed for $c \sigma=O(1)$, i.e. $\sigma=O\left(N^{-1}\right)$. Therefore collecting the powers of $1 / N$ in the r.h.s. of (19) one finds that the n-th term is proportional to $1 / N^{2 g}$ with an index $g$ related to $n$ by

$$
(2 g-1)\left(1+\frac{1}{p}\right)=n+\frac{1}{p}(l+1)
$$

So, as in 't Hooft classical result, $g$ is indeed the genus of the Riemann surface under consideration. Finally we may present the result (19) in the more transparent form

$$
U_{l}(\sigma)=\sum_{g}\left\langle\tau_{n, l}\right\rangle_{g} \frac{1}{n \pi} \Gamma\left(1-\frac{1}{p}-\frac{l}{p}\right) c^{\frac{2 g-1}{p}} p^{g} \sigma^{(2 g-1)\left(1+\frac{1}{p}\right)}
$$

This generalizes the asymptotic expansion discussed above for the Airy case $(\mathrm{p}=3)$, with $l=1,2$. Again, like in the Airy case for $p=3$, the $l=-1$ term which would violate (20) with integer $n$ and $g$, is missing : all intersection numbers $\left\langle\tau_{n, l}\right\rangle$ are of NS-type.

It is interesting to note that (20) is consistent with the Riemann-Roch relation (RR) which, for $s$-marked points (punctures), reads [12]

$$
3 g-3+s=\sum_{i=1}^{s} n_{i}+(g-1)\left(1-\frac{2}{p}\right)+\frac{1}{p} \sum_{i=1}^{s} l_{i}
$$

or equivalently,

$$
(2 g-1)\left(1+\frac{1}{p}\right)=\sum_{i=1}^{s}\left(n_{i}+\frac{1}{p} l_{i}\right)+\left(1-s+\frac{1}{p}\right)
$$


For one marked point $(s=1)$, this gives, as was derived in (20)

$$
(2 g-1)\left(1+\frac{1}{p}\right)=n+\frac{1}{p}(l+1)
$$

This shows the consistency of the matrix model results with the basic geometrical results derived by Witten [12]. The same approach in which one considers several marked points allows one to show that the matrix model is consistent with this RR geometrical rule. Furthermore we shall argue later that the matrix model provides a generalization of this RR relation for half-integer values of $p$.

Finally let us quote the simplest results that one derives from (19)

$$
\begin{aligned}
& \left\langle\tau_{1,0}\right\rangle_{g=1}=\frac{p-1}{24} \\
& \left\langle\tau_{n, l}\right\rangle_{g=2}=\frac{(1+2 p)(p-1)(p-3)}{p \cdot 5 ! \cdot 4^{2} \cdot 3} \frac{\Gamma\left(1-\frac{3}{p}\right)}{\Gamma\left(1-\frac{1+l}{p}\right)} \\
& \left\langle\tau_{n, l}\right\rangle_{g=3}=\frac{(1+2 p)(p-1)(p-5)\left(8 p^{2}-13 p-13\right)}{p^{2} \cdot 7 ! 4^{3} 3^{2}} \frac{\Gamma\left(1-\frac{5}{p}\right)}{\Gamma\left(1-\frac{1+l}{p}\right)}
\end{aligned}
$$

where $n$ and $l$ have to satisfy the condition (20) which relates $n$ and $g$ at fixed $l$; otherwise the intersection numbers vanish. Up to genus $g=9$, explicit values of the intersection numbers had been obtained in [8]. Note the factors $(p-1)$ and $(2 p+1)$ in the intersection numbers for genus $g$ on which we return in Sect. 3 and show that for $g \geq 2$, these two factors are always present. In conclusion the index $l$ takes only the $(p-1)$ values $0,1, \ldots, p-2$, which correspond to NS-punctures. The R -punctures which could have occurred for $l=-1$ are absent.

\section{One Point Function for Integer and Half-Integer $p$}

Let us first repeat what was done in the previous section in a slightly different setting, more adapted to extensions to half-integer or negative values of $p$. We start as earlier from the representation of the one-point function $\left\langle\operatorname{tr} e^{\sigma M}\right\rangle$ in the scaling limit, given by (13). The integral is around a contour in the u-plane which will depend upon the asymptotic domain under consideration:

$$
U(\sigma)=\frac{1}{\sigma} \int \frac{d u}{2 i \pi} e^{C\left(\left(u-\frac{1}{2} \sigma\right)^{p+1}-\left(u+\frac{1}{2} \sigma\right)^{p+1}\right)} .
$$

For half integer $p$, the exponent has a branch cut, and the expansion in powers of $\sigma$ gives an infinite series. For half integral $p$, we perform the change of variable $u$ to $y$

$$
u=\frac{i}{2}\left(y^{2}-y^{-2}\right), \quad d u=i\left(\frac{1}{y^{3}}+y\right) d y
$$

which will turn out to be better suited to discuss half-integer $p$. The one point function $U(\sigma)$ of (26) becomes

$$
U(\sigma)=\frac{i}{2} \oint \frac{d y}{2 i \pi}\left(y+\frac{1}{y^{3}}\right) e^{C\left(\frac{i \sigma}{4}\right)^{p+1} \frac{1}{y^{2 p+2}}\left[\left(y^{2}-i\right)^{2 p+2}-\left(y^{2}+i\right)^{2 p+2}\right]}
$$

The power $(2 p+2)$ in $(28)$ is twice the $(p+1)$ in $(26)$ and it makes the continuation to half-integers $p$ easier. (This formulation for half-integer $p$ is related to the spin structure, in 
which the double covering for a half-integer spin is related to the spin moduli space $\tilde{M}_{g}$ as shown in [37].) The integral over $y$ may lead to both NS and R-punctures. As we will see the NS type is described as integrals along cuts in the $t$-plane with $u=t^{\frac{1}{p}}$ in (19). In addition the pole at $y=0$, provides a non-vanishing residue, which corresponds to a singularity of R-type.

The one point correlation function is now written

$$
U(\sigma)=\frac{i}{2} \int \frac{d y}{2 i \pi}\left(\frac{1}{y^{3}}+y\right) g(y)
$$

with

$$
g(y)=\exp \left[C\left(\frac{i}{2}\right)^{p+1}\left(\frac{\sigma}{2}\right)^{p+1} \frac{1}{y^{2 p+2}}\left(\left(y^{2}-i\right)^{2 p+2}-\left(y^{2}+i\right)^{2 p+2}\right)\right]
$$

Let us specify this factor for a few simple values of $p$.

(i) positive integer $\mathrm{p}=1,2$

$$
\begin{aligned}
& g(y)=\exp \left[i \frac{1}{2} C \sigma^{2}\left(y^{2}-y^{-2}\right)\right] \quad(p=1) \\
& g(y)=\exp \left[-C \sigma^{3} \frac{1}{16}\left(3 y^{4}-10+3 \frac{1}{y^{4}}\right)\right] \quad(p=2)
\end{aligned}
$$

(ii) negative integer $\mathrm{p}=-1,-2,-3$

$$
\begin{aligned}
& g(y)=\exp \left[C \sigma^{-1} \frac{16 y^{4}}{\left(1+y^{4}\right)}\right] \quad(p=-2) \\
& g(y)=\exp \left[-128 i C \sigma^{-2} \frac{y^{10}-y^{6}}{\left(1+y^{4}\right)^{4}}\right] \quad(p=-3)
\end{aligned}
$$

The case of $p=-1$ is obtained from a limit $p \rightarrow-1$ in (30), noting that the constant $\mathrm{c}$, given by (18), $c=\xi /(p+1)$,

$$
g(y)=\left(\frac{y^{2}+i}{y^{2}-i}\right)^{2 \xi}, \quad(p=-1)
$$

(iii) positive half integer $p=\frac{1}{2}, \frac{3}{2}$

$$
\begin{aligned}
& g(y)=\exp \left[C(i)^{1 / 2} \frac{1}{4} \sigma^{\frac{3}{2}}\left(3 y-\frac{1}{y^{3}}\right)\right]\left(p=\frac{1}{2}\right) \\
& g(y)=\exp \left[C(i)^{3 / 2} \frac{1}{16} \sigma^{\frac{5}{2}}\left(5 y^{3}-\frac{10}{y}+\frac{1}{y^{5}}\right)\right] \quad\left(p=\frac{3}{2}\right)
\end{aligned}
$$

(iv) negative half integer $p=-\frac{1}{2},-\frac{3}{2}$

$$
\begin{aligned}
& g(y)=\exp \left[-C(i)^{\frac{3}{2}} \frac{\sigma^{\frac{1}{2}}}{y}\right] \quad\left(p=-\frac{1}{2}\right) \\
& g(y)=\exp \left[4 C(i)^{\frac{1}{2}} \sigma^{-\frac{1}{2}}\left(\frac{y}{1+y^{4}}\right)\right] \quad\left(p=-\frac{3}{2}\right)
\end{aligned}
$$

(i) positive integer $p$ We now return to (29) with positive integer $p$ to check that we recover in this new setting the results of Sect. 2. Let us start with the pure Gaussian case $(p=1)$ 
which reads from (31) and (29), with $c=\frac{i}{2} C$,

$$
U(\sigma)=\oint \frac{d y}{2 i \pi}\left(\frac{1}{y^{3}}+y\right) e^{c \sigma^{2}\left(y^{2}-y^{-2}\right)}
$$

This is just the contour integral of a total derivative and therefore it vanishes. In particular there are no terms which are proportional to $\sigma^{n}$ in $U(\sigma)$, i.e. R-type.

To summarize in the trivial $p=1$ case $\left\langle\tau_{-2,-1}\right\rangle_{g=0}=0$. For $g \geq 1$, there is no contribution, thus all intersection numbers vanish $\left\langle\tau_{n, l}\right\rangle_{g}=0$. We thus expect that the intersection numbers should involve a factor $(p-1)$. The presence of this factor had been already checked for the intersection numbers up to $g=9$ [8].

For integer $p>1$, similarly the integral (29) over a circle vanishes. However again the presence of the exponent $u^{p}$ implies different asymptotic behaviors in different regions, as discussed at length in the previous section. In this formalism with integral (29) over $y$, let us look for instance at the $p=2$ case with, from (31),

$$
g(y)=\exp \left[-a\left(y^{4}+\frac{1}{y^{4}}\right)\right]
$$

with $a=-6 c\left(\frac{\sigma}{2}\right)^{3}$ and thus

$$
U(\sigma)=\int d y\left(y+\frac{1}{y^{3}}\right) e^{-a\left(y^{4}+\frac{1}{y^{4}}\right)}
$$

Along the line at $\pi / 4$ this yields after change $y^{4} \rightarrow t$

$$
\begin{aligned}
& \int_{0}^{\infty} d t\left(t^{-\frac{1}{2}}+t^{-\frac{3}{2}}\right) e^{-a\left(t+\frac{1}{t}\right)} \\
& =\frac{1}{4 a^{\frac{1}{2}}} e^{\frac{10}{3} a}\left[\Gamma\left(\frac{1}{2}\right)+a \Gamma\left(-\frac{1}{2}\right)-a^{2} \Gamma\left(-\frac{1}{2}\right)-a^{2} \Gamma\left(-\frac{3}{2}\right)+O\left(a^{3}\right)\right]
\end{aligned}
$$

This expansion contains, as seen earlier, only NS terms, and it agrees with (19).

If we could not find any R-puncture in $\left\langle\tau_{n,-1}\right\rangle$ for positive integer $p$, it will be shown that it exists for (ii) negative values of $p$ and also for (iii),(iv) half-integer $p$. We consider halfinteger $p=-\frac{1}{2},-\frac{3}{2}, \ldots$ as well as negative integers $p=-1,-2,-3, \ldots$ in the following. (ii) negative integer $p$ Let us examine the consequence of the previous formulae if we extend them to negative values of $p$. First the connection between $n$ and $g$ established in (22)

$$
(2 g-1)\left(1+\frac{1}{p}\right)=n+\frac{1}{p}(l+1)
$$

may be extended to negative integer $p$. We have to specify the spin-index $l$ which characterizes the integration sectors and takes now $|p|$ different values,

$$
l=0,-1,-2,-3, \ldots, p+1 \quad(p \leq-1) .
$$

The R-type is still associated with $l=-1$. The choice of negative values of $l$ in the selection rule (40) for labelling the $|p|$ sectors, is reasonable since $\frac{l}{p}$ remains positive.

(ii-a) $p=-1$ case Since this case is a limit we need to return here to the initial representation (13)

$$
U(\sigma)=\frac{1}{\sigma} \int \frac{d u}{2 i \pi} e^{C\left([u-\sigma / 2)^{p+1}-(u+\sigma / 2)^{p+1}\right]}
$$


with $C=\frac{N}{p^{2}-1} \sum_{i=1}^{p-1} \frac{1}{a_{i}^{p+1}}$ to obtain the limit as $p$ goes to -1 .

$$
U(\sigma)=\frac{1}{N} \int \frac{d u}{2 i \pi}\left(\frac{u-\frac{1}{2}}{u+\frac{1}{2}}\right)^{N}
$$

Note that $\sigma$ is scaled out, and $U(\sigma)$ is a constant independent of $\sigma$. This fact is consistent with (20), where $n=1, l=0$ and $p=-1$, so that the $\sigma$ dependence becomes $\sigma^{n+\frac{1}{p}(1+l)}=\sigma^{0}$. Also note that in the limit $p \rightarrow-1$, the integral becomes (43), since $C$ involves a $\frac{1}{p+1}$ factor.

Writing $\left(u-\frac{1}{2}\right) /\left(u+\frac{1}{2}\right)=e^{-z}$ and $u=\left(1+e^{-z}\right) /\left(1-e^{-z}\right)$, we obtain

$$
U(\sigma)=-\frac{1}{N} \int_{0}^{\infty} \frac{d z}{2 \pi} \frac{e^{-z}}{\left(1-e^{-z}\right)^{2}} e^{-N z}
$$

This integral diverges linearly at the origin and a regularization is needed. As argued below this divergence is in fact linked to genus zero and after a zeta-regularization by the genus, the divergent genus zero term is discarded. Then one finds

$$
U(\sigma)=\int_{0}^{\infty} \frac{d z}{2 \pi}\left(\sum B_{n} \frac{y^{n-1}}{n !}\right) e^{-N z}=1-\frac{1}{2 N}+\frac{1}{12 N^{2}}-\frac{1}{120 N^{4}}+\cdots
$$

where the $B_{n}$ are Bernoulli numbers $\frac{t}{1-e^{-t}}=\sum_{0}^{\infty} \frac{B_{n}}{n !} t^{n}$. For this $p=-1$ case, there is no $\sigma$ dependence, and instead we have considered the dependence in the size $N$ of the matrix. This leads to the Euler characteristics $[6,14,15]$,

$$
\chi\left(\bar{M}_{g, 1}\right)=\zeta(1-2 g)=-\frac{1}{2 g} B_{2 g}
$$

Note that for $p=-1$ we have no choice other than $l=0$ in the list (41), we have the unique choice $l=0$ from (40).

(ii-b) $\mathrm{p}=-2$ We are dealing with

$$
U(\sigma)=\int d y\left(y+\frac{1}{y^{3}}\right) e^{-\frac{y^{4}}{\sigma\left(1+y^{4}\right)}} d y=\frac{1}{\sqrt{\sigma}} \int d x \frac{1}{x^{2}} e^{-\frac{4 x^{2}}{1+\sigma x^{2}}}
$$

where the equality is obtained first by noting the invariance of the integral under $y \rightarrow 1 / y$ and then $y^{2} \rightarrow \sqrt{\sigma} x$. This model is in fact equivalent to a unitary matrix model as was shown in [19]. Then the expansion of the integral (47) provides

$$
U(\sigma)=-\frac{1}{2 \sqrt{\pi \sigma}}\left(\frac{1}{8} \sigma+\frac{3^{2} \sigma^{2}}{3 ! 2^{7}}+\frac{3^{2} \cdot 5^{2}}{5 ! 2^{9}} \sigma^{3}+\frac{3^{2} \cdot 5^{2} \cdot 7^{2}}{21 \cdot 2^{18}} \sigma^{4}+\cdots\right) .
$$

It reproduces the results of the unitary matrix model. For the unitary matrix model, there are terms which come from the angular measure which yields a logarithmic potential. The result of (47) is obtained from the unitary matrix model with the coefficient $m$ of logarithmic potential in the limit $m \rightarrow 0$ [19]. We find $l=0$, and the expansion provides terms of the form $\sigma^{g-\frac{1}{2}}$ for genus $g=1,2,3, \ldots$. The intersection numbers are $\left\langle\tau_{n, 0}\right\rangle_{g}$, which are all of NS type. There is no R-type for the one point function. The result (48) gives the intersection numbers,

$$
\left\langle\tau_{1,0}\right\rangle_{g=1}=-\frac{1}{8}, \quad\left\langle\tau_{2,0}\right\rangle_{g=2}=\frac{1}{2^{7}}, \ldots
$$

which agrees with the general expression (25) for the intersection numbers of $p$ spin curves. 
(ii-c) $\mathrm{p}=-3$ The possible values of $l$ are $l=-1,0,1$. The one point function $U(\sigma)$ becomes after the scaling $y=\sigma^{\frac{1}{3}} x$,

$$
U(\sigma)=\int d x\left(\frac{\sigma^{-\frac{2}{3}}}{x^{3}}+\sigma^{\frac{2}{3}} x\right) e^{-a \frac{x^{6}\left(1-\sigma^{4 / 3} x^{4}\right)}{\left(1+\sigma^{4 / 3} x^{4}\right)^{4}}}
$$

From the selection rule relative to $p=-3$, the possible intersection numbers are $\left\langle\tau_{1,0}\right\rangle_{g=1},\left\langle\tau_{2,-1}\right\rangle_{g=2},\left\langle\tau_{4,1}\right\rangle_{g=3},\left\langle\tau_{5,0}\right\rangle_{g=4}, \ldots$ in which only $l=-1$ is of R-type. We have from (50),

$$
U(\sigma)=-\frac{1}{3} \sigma^{-\frac{2}{3}}+\frac{5}{18} \sigma^{\frac{2}{3}} \Gamma\left(\frac{1}{3}\right)+16 \sigma^{2}+\cdots
$$

and thus

$$
\left\langle\tau_{0,1}\right\rangle_{g=0}=-\frac{1}{3}, \quad\left\langle\tau_{1,0}\right\rangle_{g=1}=\frac{5}{18}, \quad\left\langle\tau_{2,-1}\right\rangle_{g=2}=16, \ldots
$$

the last one being of R-type $(l=-1)$ For the case of negative even integers $(p=-2 m)$, the selection rule (40) forbids $l=-1$ since $\frac{(2 g-1)}{p}$ is a half-integer. Therefore we have no possibility of R-puncture when $p$ is an even negative integer, whereas it does occur for odd negative integers with $l=-1$.

(iii) positive half-odd integer case $p \in \frac{1}{2}+Z$ The possible values of $l$ are now $2 p$ instead of $p$ since we made the change of variables (27) which doubles the number of sectors. This leaves the possibilities of

$$
l=-1,-\frac{1}{2}, 0, \frac{1}{2}, \ldots, p-\frac{3}{2}
$$

(iii-a) $\mathrm{p}=\frac{1}{2}$ For $p=\frac{1}{2}$, the only possible value is $l=-1$, which is an R-puncture. We have from (34), the one point function,

$$
U(\sigma)=\frac{i}{2} \oint \frac{d y}{2 i \pi}\left(\frac{1}{y^{3}}+y\right) e^{c^{\prime} \sigma^{\frac{3}{2}}\left(3 y-\frac{1}{y^{3}}\right)}
$$

where the above integral runs over a contour around $y=0$, and $c^{2}=\frac{i}{2} c^{2}$. The possible value of the spin component $l$ is only $l=-1$ from (53). Indeed we have the selection rule for the intersection numbers in (20) $\sigma^{3(2 g-1)}=\sigma^{n+2(l+1)}$ with $l=-1$, which is integer power of $\sigma$. Hence the intersection numbers $\left\langle\tau_{n, l}\right\rangle_{g}$ are with $n=3+6 m(m=1,2, \ldots)$. Non vanishing possible intersection numbers are $\left\langle\tau_{3,-1}\right\rangle_{g=1},\left\langle\tau_{9,-1}\right\rangle_{g=2},\left\langle\tau_{15,-1}\right\rangle_{g=3}, \ldots$. These values may be obtained, after a Taylor expansion of the second exponential term, and picking up the residues at the $y=0$ pole. This one point is easily computed in closed form by the same technique,

Thus we have

$$
U(\sigma)=\frac{i}{2} \sum_{j=0}^{\infty} c^{\prime 4 j+2} \frac{3^{2+3 j}}{j !(3 j+3) !}(-1)^{j} \sigma^{3+6 j}
$$

These terms are consistent with the selection rule, since the $g$ dependence in $\sigma$ is $\sigma^{3(2 g-1)}$. Using the expression of $U(\sigma)$ of (55), we have an explicit result in closed form for

$$
T(z)=\int_{0}^{\infty} U(\sigma) \sigma^{4} e^{-\sigma^{2} / z} d \sigma=\frac{9}{4} i z^{4} e^{27 c^{\prime 4} z^{3}}
$$


This equation shows the relation between $p=\frac{1}{2}$ and the Airy distribution $(p=2)$ with a logarithmic term.

(iii-b) $\mathrm{p}=\frac{3}{2}$ This spin $p=\frac{3}{2}$ may correspond to a Rarita-Schwinger operator $[37,38]$.

The possible values of $l$ are $l=-1,-\frac{1}{2}, 0$. We have

$$
U(\sigma)=\oint \frac{d y}{2 \pi i}\left(\frac{1}{y^{3}}+y\right) e^{c\left(\frac{\sigma}{2}\right)^{\frac{5}{2}}\left[5 y^{3}-\frac{10}{y}+\frac{1}{y^{5}}\right]}
$$

where the contour runs around $y=0$. This gives terms of order $\sigma^{\frac{5}{3}}, \sigma^{5}, \sigma^{\frac{25}{3}}, \ldots$, which are consistent with the selection rule for $p=\frac{3}{2}$. The expansion of $U(\sigma)$ gives the series of terms of $\left\langle\tau_{n, l}\right\rangle_{g} \sigma^{n+\frac{2}{3}(l+1)}$, where the coefficients, i.e. intersection numbers, become $\left\langle\tau_{-2,-\frac{1}{2}}\right\rangle_{g=0}$, $\left\langle\tau_{1,0}\right\rangle_{g=1},\left\langle\tau_{5,-1}\right\rangle_{g=2},\left\langle\tau_{8,-\frac{1}{2}}\right\rangle_{g=3}$. The term $l=-1$ which gives an integer power of $\sigma$ is of R-type. At higher orders the R-type appears with $\sigma^{5}, \sigma^{15}, \sigma^{25}, \ldots$. These terms of integer power $\sigma^{5+10 m}, m=0,1,2,3, \ldots$ are obtained from the contour integral along the circle $|y|=1$ where the pole at $y=0$ in (57) exists, which are evaluated as

$$
U_{R}(\sigma)=\frac{5}{2^{5}} c^{2} \sigma^{5}-\frac{7 \cdot 5^{4}}{3 \cdot 2^{16}} c^{6} \sigma^{15}+\frac{79 \cdot 11 \cdot 5^{7} \cdot 3^{2}}{10 ! \cdot 2^{24}} c^{10} \sigma^{25}+\cdots
$$

where the first term is $g=2$, and the second term is a $g=5$ contribution to a R-puncture.

The NS-type intersection numbers are obtained from (57) in the sector defined by the replacement $y^{3}=t$, and with $a=-c\left(\frac{\sigma}{2}\right)^{5 / 2}$.

$$
\begin{aligned}
U_{N S}(\sigma) & =\frac{1}{3} \int_{0}^{\infty} \frac{d t}{2 \pi} t^{-\frac{2}{3}}\left(t^{\frac{1}{3}}+\frac{1}{t}\right) e^{-a\left(5 t-10 t^{-1 / 3}+t^{-5 / 3}\right)} \\
& =\frac{1}{3 a} \int_{0}^{\infty} \frac{d t}{2 \pi} a^{\frac{2}{3}} t^{-\frac{2}{3}}\left(\left(\frac{t}{a}\right)^{1 / 3}+\frac{a}{t}\right) e^{-5 t}\left(1+\frac{10}{t^{1 / 3}} a^{4 / 3}+\left(\frac{50}{t^{2 / 3}}-\frac{1}{t^{5 / 3}}\right) a^{8 / 3}+\cdots\right)
\end{aligned}
$$

It provides a series expansion,

$$
\begin{aligned}
& U_{N S}(\sigma)=\frac{1}{15 a} \int_{0}^{\infty} \frac{d x}{2 \pi}\left(a^{1 / 3}\left(\frac{x}{5}\right)^{-1 / 3}+a^{5 / 3}\left(\frac{x}{5}\right)^{-5 / 3}\right) e^{-x}\left(1+\frac{10 a^{4 / 3}}{\left(\frac{x}{5}\right)^{1 / 3}}+\cdots\right) \\
& =\frac{5^{1 / 3}}{15} \Gamma\left(\frac{2}{3}\right)\left(-c\left(\frac{\sigma}{2}\right)\right)^{-\frac{5}{3}}+\frac{5^{5 / 3}}{15} \Gamma\left(-\frac{2}{3}\right)\left(-c\left(\frac{\sigma}{2}\right)\right)^{\frac{5}{3}}+\cdots
\end{aligned}
$$

This series gives terms with non-integer powers of $\sigma$, thus of NS type, which are consistent with the selection rule for $p=\frac{3}{2}$. Indeed the series (60) shows non-vanishing intersection numbers $\left\langle\tau_{-2,-\frac{1}{2}}\right\rangle_{g=0},\left\langle\tau_{1,0}\right\rangle_{g=1},\left\langle\tau_{5,-1}\right\rangle_{g=2}, \ldots$. which were expected from the selection rule $\sigma^{(2 g-1)\left(1+\frac{1}{p}\right)}=\sigma^{n+\frac{1}{p}(l+1)}$.

The case of $p=\frac{3}{2}$ is particularly interesting since the level $k$ of $s u(2),(k=p-2)$, becomes $k=-\frac{1}{2}$. The $\mathcal{N}=2$ super symmetric $\widehat{s u}(2)_{-\frac{1}{2}}$ WZW model is a non-unitary conformal field theory with central charge $c=3-\frac{6}{k+2}=-1$ [22,24], and for the coset $\widehat{s u}(2)_{-1 / 2} / u(1)$ WZW model, the central charge $c=-2$. The $\widehat{s u}(2)$ means the affine super algebra [22].

(iv) negative half-odd integer $p$ For negative half-odd integers $p$, there are $2|p|$ allowed values of $l$, namely $l=-1,-\frac{1}{2}, 0, \frac{1}{2}, 1, \frac{3}{2}, \ldots,|p|-\frac{3}{2}$.

(iv-a) $p=-\frac{1}{2}$ case 
Since for $p=-\frac{1}{2}$ the factor $\frac{1}{p}(l+1)$ becomes an integer, the $\sigma$ dependence does not involve fractional powers. Thus the $p=-\frac{1}{2}$ is interpretated as R-type punctures.

$U(\sigma)$ has here the expression

$$
U(\sigma)=\int d y\left(\frac{1}{y^{3}}+y\right) e^{c \sqrt{\sigma} \frac{1}{y}}
$$

where the y-integral runs on a contour around $y=0$. Expanding in $\sigma$ the contour integral, the only non-vanishing term is $\frac{c^{2}}{2} \sigma$. Since the intersection number $\left\langle\tau_{n, l}\right\rangle$ is the coefficient of the term $\sigma^{n+\frac{1}{p}(l+1)}\left(=\sigma^{(2 g-1)\left(1+\frac{1}{p}\right)}\right)$ in the expansion of $U(\sigma)$, this term corresponds to $n=1, l=-1$, with $g=0$. This corresponds to an R-puncture $(l=-1)$ for $g=0$ with non-zero $\left\langle\tau_{1,-1}\right\rangle_{g=0}$.

The first term $\frac{1}{y^{3}}$ does not contribute to the pole at $y=0$. Given the essential singularity at the origin one has to be more careful.

Indeed if one changes $y \rightarrow-\frac{1}{x}$, the first term of the integral becomes

$$
\int_{0}^{\infty} d x x e^{-c \sqrt{\sigma} x}=\frac{1}{c^{2} \sigma}
$$

which could not be found in a $\sigma$ expansion. This result corresponds to a term $\sigma^{n+\frac{l+1}{p}}$ with $p=-\frac{1}{2}, n=-1, l=-1$. This leads to a non-zero intersection number $\left\langle\tau_{-1,-1}\right\rangle_{g=1}$. The expansion has just two terms of order $\sigma$ and $\sigma^{-1}$, and there are no more terms. This is consistent with the factor $(2 p+1)$ in the intersection numbers for general $p$ (as shown in (19) [8]), which vanishes for $p=-\frac{1}{2}$.

(iv-b) $\mathrm{p}=-\frac{3}{2}$ For $p=-\frac{3}{2}$, there are $2|p|$ allowed values for $l$ are $l=-1,-\frac{1}{2}, 0$ and the selection rule for $g, n, l$ reads

$$
\sigma^{(2 g-1)\left(1+\frac{1}{p}\right)}=\sigma^{(2 g-1) \frac{1}{3}}=\sigma^{n-\frac{2}{3}(l+1)}
$$

Therefore for $g=0$, it gives the power $\sigma^{-\frac{1}{3}}$, which leads to $n=0, l=-\frac{1}{2}$, i.e. $\left\langle\tau_{0,-\frac{1}{2}}\right\rangle_{g=0}$. For $g=1$, it becomes $\sigma^{\frac{1}{3}}$, and we obtain $n=1, l=0$, i.e. $\left\langle\tau_{1,0}\right\rangle_{g=1}$. For $g=2$, we $\sigma^{(2 g-1)\left(1+\frac{1}{p}\right)}=\sigma^{(2 g-1) \frac{1}{3}}=\sigma$. Thus we have $n=1, l=-1,\left\langle\tau_{1,-1}\right\rangle_{g=2}$, an R-type case.

For $g=3$, we have $\sigma^{(2 g-1) \frac{1}{3}}=\sigma^{\frac{5}{3}}$, then $n=2, l=-\frac{1}{2},\left\langle\tau_{2,-\frac{1}{2}}\right\rangle_{g=3}$.

For $g=4$, we have similarly $\left\langle\tau_{3,0}\right\rangle_{g=4}$ with $\sigma^{\frac{7}{3}}$. For $g=5$, we have $\sigma^{3}$ and $\left\langle\tau_{3,-1}\right\rangle_{g=5}$. This is again an R-type. Thus we find R-punctures at $g=2+3 m,(m=0,1,2, \ldots)$, for which $U(\sigma)$ yields integer powers $\sigma^{2 m+1}$, and the intersection numbers are

$$
\left\langle\tau_{2 m+1,-1}\right\rangle_{g=2+3 m}, \quad(m=0,1,2, \ldots)
$$

We can verify these results by the explicit calculation of (29).

We take $c 2^{\frac{1}{2}} \sigma^{-\frac{1}{2}}=\sigma^{\prime-\frac{1}{2}}$ for simplicity. The one point function is

$$
U(\sigma)=\oint d y\left(\frac{1}{y^{3}}+y\right) \exp \left(-\sigma^{\prime-\frac{1}{2}} \frac{y}{1+y^{4}}\right)
$$

where the integral runs around $y=0$. This integral gives an expansion in integer powers of $\sigma^{\prime}$ if we rescale $y$ by $\sigma^{\prime 1 / 2} y$. This leads to R-intersection numbers with a $\left.\sigma^{2 m+1}(m=0,1,2, \ldots)\right)$ dependence. 
When we change $y \rightarrow \sigma^{\prime-\frac{1}{6}} t^{-\frac{1}{3}}$ in (65), we obtain

$$
\begin{aligned}
U(\sigma) & =-\frac{1}{3} \int d t\left(\sigma^{\prime-\frac{1}{3}} t^{-\frac{5}{3}}+\sigma^{\prime \frac{1}{3}} t^{-\frac{1}{3}}\right) \exp \left(-\frac{t}{1+\sigma^{\prime \frac{2}{3}} t^{\frac{4}{3}}}\right) \\
& =\left(-\frac{1}{3}\right)\left[\sigma^{\prime-\frac{1}{3}} \Gamma\left(-\frac{2}{3}\right)+\frac{5}{2} \sigma^{\prime \frac{1}{3}} \Gamma\left(\frac{2}{3}\right)+3 \sigma^{\prime}+O\left(\sigma^{\prime \frac{5}{3}}\right)\right]
\end{aligned}
$$

The integral of $y$ is from 0 to $\infty$, and it leads to gamma function. The expansion of this integral for small $\sigma^{\prime}$ gives terms of order $\sigma^{\frac{1}{3}(2 g-1)},(\mathrm{g}=0,1,2, \ldots)$. This expansion is consistent with the result of the selection rule (63). Thus we have an R-puncture $l=-1$, with integer powers of $\sigma$, for $g=2+3 m,(m=0,2,3, \ldots)$. The dependence in $\sigma$ for the R-type reads $\sigma^{(2 g-1) \frac{1}{3}}=\sigma^{(2 m+1)}$ with $g=2+3 m$, which is consistent with the R-calculation of the integral (65). We also find NS-types with fractional powers of $\sigma$ by (66). This analysis may be easily extended to $p=-\frac{7}{2},-\frac{9}{2}, \ldots$. , and we find both R $(l=-1)$ and NS $(l \neq-1)$ contributions.

Thus we have studied many cases provided by the representation (29) of the one point function. We have found R and NS-punctures in those cases. The computation of the intersection numbers of R-punctures for $p=\frac{1}{2}, \frac{3}{2}, \ldots$ results from a contour integral around the pole at $y=0$. For negative integers and negative half-integers, the intersection numbers are obtained by the same technique, and they yield both R and NS- punctures.

\section{Two Point Correlation Function $U\left(\sigma_{1}, \sigma_{2}\right)$}

Higher correlation function, involving the intersection numbers which appear as coefficients of several $\bar{t}_{n}$ in the expansion of the free energy, can be handled by the same technique. Again one considers in the $1 / \lambda_{a}$ expansion of $\left\langle\prod_{a=1}^{K} \operatorname{det}\left(1-\frac{M}{\lambda_{a}}\right)\right\rangle$ the coefficients which involve $\left\langle\operatorname{trM}^{\mathrm{n}_{1}} \operatorname{trM}^{\mathrm{n}_{2}} \cdots\right\rangle$. A generating function for these coefficients are the $U\left(\sigma_{1}, \cdots, \sigma_{n}\right)$. Let us consider the two point function

$$
U\left(\sigma_{1}, \sigma_{2}\right)=\left\langle\operatorname{tr} e^{\sigma_{1} M} \operatorname{tr} e^{\sigma_{2} M}\right\rangle .
$$

It is given by the formula (10) for $n=2$,

$$
U\left(\sigma_{1}, \sigma_{2}\right)=e^{\sum_{1}^{n} \sigma_{i}^{2}} \oint \prod_{i=1}^{2} \frac{d u_{i}}{2 i \pi} e^{\sum_{1}^{n} u_{i} \sigma_{i}} \prod_{\alpha=1}^{N} \prod_{i=1}^{2}\left(1-\frac{\sigma_{i}}{a_{\alpha}-u_{i}}\right) \operatorname{det} \frac{1}{u_{i}-u_{j}+\sigma_{i}}
$$

The diagonal part of the determinant gives the disconnected part $\left\langle\operatorname{tr} e^{\sigma_{1} M}\right\rangle,\left\langle\operatorname{tr} e^{\sigma_{2} M}\right\rangle$. The off -diagonal term gives the connected correlator which, after the shift $u_{1} \rightarrow u_{1}-\frac{1}{2} \sigma_{1}, u_{2} \rightarrow$ $u_{2}-\frac{1}{2} \sigma_{2}$, deals with a product, which is conveniently replaced by an additional integration

$$
\begin{gathered}
\frac{1}{u_{1}-u_{2}+\frac{1}{2}\left(\sigma_{1}+\sigma_{2}\right)} \frac{1}{u_{1}-u_{2}-\frac{1}{2}\left(\sigma_{1}+\sigma_{2}\right)} \\
=\frac{2}{\sigma_{1}+\sigma_{2}} \int_{0}^{\infty} d x e^{-x\left(u_{1}-u_{2}\right)} \sinh \frac{x}{2}\left(\sigma_{1}+\sigma_{2}\right)
\end{gathered}
$$

Positive integer $p$ We now return to the large-N scaling [6] which led to the $p$-spin formulae with $\sigma_{i} \sim N^{-1 /(p+1)}$, with the same scaling expansion of $\prod_{\alpha=1}^{N} \prod_{i=1}^{2}\left(1-\frac{\sigma_{i}}{a_{\alpha}-u_{i}}\right)$ for the 
source specified by the conditions (4). This yields

$$
\begin{aligned}
U_{c}\left(\sigma_{1}, \sigma_{2}\right)= & \frac{2}{\sigma_{1}+\sigma_{2}} \frac{1}{(2 i \pi)^{2}} \int_{0}^{\infty} d x \int d u_{1} d u_{2} \sinh \left(\frac{1}{2} x\left(\sigma_{1}+\sigma_{2}\right)\right) e^{-x\left(u_{1}-u_{2}\right)} \\
& \times \exp \left[-\frac{N}{p^{2}-1} c \sum_{i=1}^{2}\left(\left(u_{i}+\frac{1}{2} \sigma_{i}\right)^{p+1}-\left(u_{i}-\frac{1}{2} \sigma_{i}\right)^{p+1}\right)\right]
\end{aligned}
$$

where the $u_{1} u_{2}$ integrals are similar to the $u$-integral for the one-point function. The expression (70) has the form

$$
\begin{aligned}
U_{c}\left(\sigma_{1}, \sigma_{2}\right)= & \frac{2}{\sigma_{1}+\sigma_{2}} \frac{1}{(2 i \pi)^{2}} \int_{0}^{\infty} d x \int d u_{1} d u_{2} \sinh \left(\frac{1}{2} x\left(\sigma_{1}+\sigma_{2}\right)\right) \\
& \times e^{-x\left(u_{1}-u_{2}\right)} G\left(u_{1}, \sigma_{1}\right) G\left(u_{2}, \sigma_{2}\right)
\end{aligned}
$$

with

$$
G(u, \sigma)=\exp \left[-\frac{N}{p^{2}-1} c\left(\left(u+\frac{1}{2} \sigma\right)^{p+1}-\left(u-\frac{1}{2} \sigma\right)^{p+1}\right)\right] .
$$

These expressions may all be expanded in powers of $\sigma_{1}$ and $\sigma_{2}$ and provide a generating function of the two-point intersection numbers. The lowest order in $\sigma_{1}$ is a term of order $\sigma_{1}^{\frac{1}{p}} \sigma_{2}^{2+\frac{1}{p}}$; in agreement with (22), the RR selection rule, this gives a non-zero $\left\langle\tau_{0,0} \tau_{2,0}\right\rangle_{g=1}$

$$
\left\langle\tau_{0,0} \tau_{2,0}\right\rangle_{g=1}=\frac{p-1}{24}
$$

which is equal to $\left\langle\tau_{1,0}\right\rangle_{g=1}$ as implied by the string equation, or lowest Virasoro constraint for the equations of motion,

$$
\left\langle\tau_{0,0} \tau_{n, l}\right\rangle_{g}=\left\langle\tau_{n-1, l}\right\rangle_{g}
$$

When $p=2$, we find

$$
\begin{aligned}
U\left(\sigma_{1}, \sigma_{2}\right) & =\frac{2}{\left(\sigma_{1}+\sigma_{2}\right) \sqrt{\sigma_{2}}} e^{\frac{1}{24}\left(\sigma_{1}^{3}+\sigma_{2}^{3}\right)} \int_{0}^{\infty} d x \sinh \left(x \frac{\sqrt{\sigma_{1}}}{2}\left(\sigma_{1}+\sigma_{2}\right)\right) e^{-\frac{\sigma_{1}+\sigma_{2}}{\sigma_{2}} x^{2}} \\
& =\frac{1}{\sigma_{1}+\sigma_{2}} e^{\frac{1}{24 N^{\prime 2}}\left(\sigma_{1}+\sigma_{2}\right)^{3}} \sum_{m=0}^{\infty} \frac{(-1)^{m}}{m !(2 m+1)}\left(\frac{\sigma_{1} \sigma_{2}\left(\sigma_{1}+\sigma_{2}\right)}{8}\right)^{m} \sqrt{\sigma_{1} \sigma_{2}} \\
& =\sum_{n_{1}, n_{2}}\left\langle\tau_{n_{1}, 0} \tau_{n_{2}, 0}\right\rangle_{g} \sigma_{1}^{n_{1}+\frac{1}{2}} \sigma_{2}^{n_{2}+\frac{1}{2}}
\end{aligned}
$$

Thus we have obtained explicit formulae for the intersection numbers $\left\langle\tau_{n_{1}, o} \tau_{n_{2}, 0}\right\rangle_{g}$ at arbitrary genus $g$. The above formula for $p=2$ may be expressed in a symmetric way as

$$
U_{c}\left(\sigma_{1}, \sigma_{2}\right)=\frac{2}{\left(\sigma_{1}+\sigma_{2}\right)} e^{\frac{1}{24}\left(\sigma_{1}^{3}+\sigma_{2}^{3}\right)} \int_{0}^{\infty} d x \sinh \left(x \frac{\sqrt{\sigma_{1} \sigma_{2}}}{2}\left(\sigma_{1}+\sigma_{2}\right)\right) e^{-\left(\sigma_{1}+\sigma_{2}\right) x^{2}}
$$

This does not yield any integer power of the $\sigma_{i}, \operatorname{since} \sinh (x)$ is odd : the powers of $\sigma_{i}$ $(i=1,2)$ are all half-integers. Hence the two-puncture points are of NS-type, no R-puncture for $p=2$. 
For $p=3$, similarly from the explicit expression for the two-point function [44], one does not find R-punctures. Indeed for $p=3$, we have [44],

$$
\begin{aligned}
U_{c}\left(\sigma_{1}, \sigma_{2}\right)= & \frac{2}{\left(\sigma_{1}+\sigma_{2}\right)\left(3 \sigma_{2}\right)^{1 / 3}} \int_{0}^{\infty} d x \sinh \left(\frac{\sigma_{1}+\sigma_{2}}{2}\left(3 \sigma_{1}\right)^{1 / 3} x\right) A_{i}\left(x-\frac{1}{4 \cdot 3^{1 / 3}} \sigma_{1}^{8 / 3}\right) \\
& \times A_{i}\left(-\left(\frac{\sigma_{1}}{\sigma_{2}}\right)^{1 / 3} x-\frac{1}{4 \cdot 3^{1 / 3}} \sigma_{2}^{8 / 3}\right)
\end{aligned}
$$

where the Airy function $A_{i}(x)$ is defined by

$$
A_{i}(x)=\int_{-\infty}^{\infty} \frac{d u}{2 \pi} e^{\frac{i}{3} u^{3}+i u x}
$$

The above formula may be written in a symmetric way as

$$
\begin{aligned}
U_{c}\left(\sigma_{1}, \sigma_{2}\right)= & \frac{2}{\left(\sigma_{1}+\sigma_{2}\right)} \int_{0}^{\infty} d x \sinh \left(\frac{\sigma_{1}+\sigma_{2}}{2}\left(3 \sigma_{1}\right)^{1 / 3}\left(3 \sigma_{2}\right)^{1 / 3} x\right) \\
& \times A_{i}\left(\left(3 \sigma_{2}\right)^{1 / 3} x-\frac{1}{4 \cdot 3^{1 / 3}} \sigma_{1}^{8 / 3}\right) A_{i}\left(-\left(3 \sigma_{1}\right)^{1 / 3} x-\frac{1}{4 \cdot 3^{1 / 3}} \sigma_{2}^{8 / 3}\right)
\end{aligned}
$$

Note that the Airy function $A_{i}(x)$ decays exponentially for $x \rightarrow+\infty$, and oscillates when $x \rightarrow-\infty$. Therefore the integral over $x$ is finite. The $p=3$ intersection numbers for two points are all of Neveu-Schwarz type. More details for this $p=3$ case may be found in [44].

For $p=4$, we have similarly

$$
\begin{aligned}
U_{c}\left(\sigma_{1}, \sigma_{2}\right)= & \frac{2}{\left(\sigma_{1}+\sigma_{2}\right)\left(4 \sigma_{2}\right)^{1 / 4}} \int_{0}^{\infty} d x \int_{0}^{\infty} d v_{1} d v_{2} \sinh \left(\frac{\sigma_{1}+\sigma_{2}}{2}\left(4 \sigma_{1}\right)^{1 / 4} x\right) \\
& \times e^{-\frac{\sigma_{1}^{3}}{2}\left(\frac{1}{4 \sigma_{1}}\right)^{1 / 2} v_{1}^{2}-\frac{\sigma_{2}^{3}}{2}\left(\frac{1}{4 \sigma_{2}}\right)^{1 / 2} v_{2}^{2}} e^{-\frac{1}{4} v_{1}^{4}+x v_{1}-\frac{1}{4} v_{2}^{4}-a x v_{2}}
\end{aligned}
$$

where $a=\left(\sigma_{1} / \sigma_{2}\right)^{1 / 4}$. This may be written as

$$
\begin{aligned}
U_{c}\left(\sigma_{1}, \sigma_{2}\right)= & \frac{2}{\left(\sigma_{1}+\sigma_{2}\right)} \int_{0}^{\infty} d x \sinh \left(\frac{\sigma_{1}+\sigma_{2}}{2}\left(4 \sigma_{1}\right)^{1 / 4}\left(4 \sigma_{2}\right)^{1 / 4} x\right) \\
& \times \phi_{+}(x) \phi_{-}(x)
\end{aligned}
$$

where

$$
\begin{aligned}
& \phi_{+}(x)=\int d v e^{-\frac{1}{4} v^{4}-\frac{1}{4} \sigma_{1}^{5 / 2} v^{2}+\left(4 \sigma_{2}\right)^{1 / 4} x v} \\
& \phi_{-}(x)=\int d v e^{-\frac{1}{4} v^{4}-\frac{1}{4} \sigma_{2}^{5 / 2} v^{2}-\left(4 \sigma_{1}\right)^{1 / 4} x v}
\end{aligned}
$$

The two-points intersection numbers may be obtained for larger values of $p$ by this method [44].

(ii) half-integer p Using the expression (34) of $g(y)$, the two point correlation function $U\left(\sigma_{1}, \sigma_{2}\right)$ is given by

$$
\begin{aligned}
U\left(\sigma_{1}, \sigma_{2}\right)= & \oint \frac{d y_{1} d y_{2}}{(2 \pi i)^{2}}\left(\frac{1}{y_{1}^{3}}+y_{1}\right)\left(y_{2}+\frac{1}{y_{2}^{3}}\right) g\left(y_{1}\right) g\left(y_{2}\right) \\
& \times \frac{1}{\left(\frac{\sigma_{1}}{2} u_{1}-\frac{\sigma_{2}}{2} u_{2}\right)^{2}-\frac{1}{4}\left(\sigma_{1}+\sigma_{2}\right)^{2}}
\end{aligned}
$$


where $u_{i}=\frac{i}{2}\left(y_{i}^{2}-y_{i}^{-2}\right)$ and $g\left(y_{i}\right)$ is given by (34) and (35). The double-integral is around contours circling around $y_{1}=y_{2}=0$.

(ii-a) $\mathrm{p}=\frac{1}{2}$ The $p=\frac{1}{2}$ case presents, as we have seen for the one point function in (55), a spin component $l=-1$ ). The two point function for $p=\frac{1}{2}$ is given from (84) and, after the shift $y_{i} \rightarrow \sigma_{i}^{-\frac{1}{2}} y_{i}, c^{\prime 2}=\left(\frac{i}{2}\right)^{p+1} c^{2}$, it reads

$$
\begin{aligned}
U_{c}\left(\sigma_{1}, \sigma_{2}\right)= & 4 \oint \frac{d y_{1} d y_{2}}{(2 \pi i)^{2}}\left(y_{1}+\frac{\sigma_{1}^{2}}{y_{1}^{3}}\right)\left(y_{2}+\frac{\sigma_{2}^{2}}{y_{2}^{3}}\right) e^{c^{\prime} \sigma_{1}\left(3 y_{1}-\frac{\sigma_{1}^{2}}{y_{1}^{3}}\right)+c^{\prime} \sigma_{2}\left(3 y_{2}-\frac{\sigma_{2}^{2}}{y_{2}^{3}}\right)} \\
& \times \frac{1}{\left(y_{1}^{2}-\frac{\sigma_{1}^{2}}{y_{1}^{2}}-y_{2}^{2}+\frac{\sigma_{2}^{2}}{y_{2}^{2}}\right)^{2}+4\left(\sigma_{1}+\sigma_{2}\right)^{2}}
\end{aligned}
$$

The selection rule (22), $3 g-3+s=\sum n_{i}+(g-1)\left(1-\frac{2}{p}\right)+\frac{1}{p} \sum l_{i}$, becomes with $s=2$ (two-point), $l_{i}=-1$,

$$
6 g=n_{1}+n_{2}
$$

Thus we expect the series $U\left(\sigma_{1}, \sigma_{2}\right)=\sum_{g, n_{1}, n_{2}} C_{g, n_{1}, n_{2}} c^{\prime 4 g} \sigma_{1}^{n_{1}} \sigma_{2}^{n_{2}}$ with integers $n_{1}$ and $n_{2}$, and coefficient $C_{g, n_{1}, n_{2}}$.

The two point function is expanded for small $\sigma_{i}$ as

$$
\begin{aligned}
U\left(\sigma_{1}, \sigma_{2}\right)= & 4 \oint \frac{d y_{1} d y_{2}}{(2 i \pi)^{2}}\left(y_{1}+\frac{\sigma_{1}^{2}}{y_{1}^{3}}\right)\left(y_{2}+\frac{\sigma_{2}^{2}}{y_{2}^{3}}\right) \frac{1}{\left(y_{1}^{2}-y_{2}^{2}\right)^{2}} \\
& \times \frac{1}{1-f} e^{c^{\prime} \sigma_{1}\left(3 y_{1}-\frac{\sigma_{1}^{2}}{y_{1}^{3}}\right)+c^{\prime} \sigma_{2}\left(3 y_{2}-\frac{\sigma_{2}^{2}}{y_{2}^{3}}\right)}
\end{aligned}
$$

where

$$
f=\frac{2}{y_{1}^{2}-y_{2}^{2}}\left(\frac{\sigma_{1}^{2}}{y_{1}^{2}}-\frac{\sigma_{2}^{2}}{y_{2}^{2}}\right)-\frac{1}{\left(y_{1}^{2}-y_{2}^{2}\right)^{2}}\left(\left(\frac{\sigma_{1}^{2}}{y_{1}^{2}}-\frac{\sigma_{2}^{2}}{y_{2}^{2}}\right)^{2}+4\left(\sigma_{1}+\sigma_{2}\right)^{2}\right)
$$

The factor $\frac{1}{1-f}$ is expanded as $\sum_{m=0}^{\infty} f^{m}$. The two point function $U\left(\sigma_{1}, \sigma_{2}\right)$ is expanded in the power of $\sigma_{1}, \sigma_{2}$ and $c^{\prime}$, which becomes a series of $c^{\prime 4 g} \sigma_{1}^{n_{1}} \sigma_{2}^{n_{2}}$ with $n_{1}+n_{2}=6 \mathrm{~g}(\mathrm{~g}$ is genus). Thus we first perform Taylor expansions of $c^{\prime}, \sigma_{1}$ and $\sigma_{2}$ for each fixed genus $g$. The contour integral around $y_{1}=0$ and $y_{2}=0$ depends on the order of $y_{i}(\mathrm{i}=1,2)$. For instance, genus one case of $n_{1}=2, n_{2}=4$, the integral of $y_{2}$ is firstly done, and secondly $y_{1}$ integral is evaluated, for a non vanishing result. Opposite order gives a null result. Thus the contour integral of $y_{i}$ is non-commutative. There are poles at $y_{1}= \pm y_{2}$ in the contour integral for $c^{\prime 4} \sigma_{1}^{2} \sigma_{2}^{4}$ term. Their contributions are cancelled, however. After taking all residues, we have

$$
\begin{aligned}
U\left(\sigma_{1}, \sigma_{2}\right)= & 4\left(\frac{3^{3}}{4} c^{\prime 4}\left(\sigma_{1}^{2} \sigma_{2}^{4}+\sigma_{1}^{4} \sigma_{2}^{2}\right)-\frac{3^{5}}{40} c^{\prime 8} \sigma_{1}^{6} \sigma_{2}^{6}+\frac{3^{5}}{160} c^{\prime 8}\left(\sigma_{1}^{2} \sigma_{2}^{10}+\sigma_{1}^{10} \sigma_{2}^{2}\right)\right. \\
& \left.+O\left(c^{\prime 16}\right)\right),
\end{aligned}
$$

where the residues of the term of $c^{\prime 8} \sigma_{1}^{4} \sigma_{2}^{8}$ for $y_{1}=0$ and $y_{2}=0$ have opposite signs when the order of evaluation of $y_{i}$ is changed, then sum of two terms is cancelled. The term of 
$c^{\prime 8} \sigma_{1}^{2} \sigma_{2}^{10}$ has a non-vanishing value by the order of the first $y_{2}=0$ residue, and the second $y_{1}=0$ residue. Opposite order of residue gives vanishing residue.

From the selection rule (86), the term of order of $c^{\prime 4}$ corresponds to genus one, and the term of order of $c^{\prime 8}$ to genus two. This confirms that the two-point function is described by R-punctures $\left(l_{1}=l_{2}=-1\right)$ and satisfies the selection rule (86). The values of $n_{1}$ and $n_{2}$ are even integers, and there is no contribution from odd integer $n_{i}$ in two point function $U\left(\sigma_{1}, \sigma_{2}\right)$.

(ii-b) $\mathrm{p}=\frac{3}{2}$ The selection rule becomes from (22) for $p=\frac{3}{2}$,

$$
\frac{10}{3} g=n_{1}+n_{2}
$$

when $l_{1}=l_{2}=-1$ (R type). The genus $g$ is an integer, which leads to $g=3 m(m=$ $1,2,3, \ldots)$. The factor of the exponent of (85) becomes for $p=\frac{3}{2}(i=1,2)$,

$$
g\left(\sigma_{i}^{-\frac{1}{2}} y_{i}\right)=e^{\sum_{i} c^{\prime} \sigma_{i}\left(5 y_{i}^{3}-\frac{10 \sigma_{i}^{2}}{y_{i}}+\frac{\sigma_{i}^{4}}{y_{i}^{5}}\right)}
$$

and the other terms are the same as in the expression (85). From the residues of $y_{1}=0, y_{2}=0$, the two-point function for the R type $\left(l_{1}=l_{2}=-1\right)$ is expanded in the series of $c^{\prime 4 g / 3} \sigma_{1}^{n_{1}} \sigma_{2}^{n_{2}}$ with $g=3 m, n_{1}+n_{2}=10 m(m=1,2, \ldots)$,

$$
\begin{aligned}
U_{R}\left(\sigma_{1}, \sigma_{2}\right)= & 4\left(-\frac{5^{3}}{4} c^{\prime 4}\left(\sigma_{1}^{2} \sigma_{2}^{8}+\sigma_{1}^{8} \sigma_{2}^{2}\right)-5^{3}\left(\sigma_{1}^{3} \sigma_{2}^{7}+\sigma_{1}^{7} \sigma_{2}^{3}\right)\right. \\
& \left.-\frac{5^{3} \cdot 3}{2}\left(\sigma_{1}^{4} \sigma_{2}^{6}+\sigma_{1}^{6} \sigma_{2}^{4}\right)+O\left(c^{8}\right)\right), \quad\left(p=\frac{3}{2}\right)
\end{aligned}
$$

The term of $c^{\prime 4} \sigma_{1}^{5} \sigma_{2}^{5}$ has vanishing residue at $y_{1}=y_{2}=0$ and $y_{1}= \pm y_{2}$. This expansion is consistent with the selection rule for $g=3 m,(m=1,2,3, \ldots)$ and $\frac{10}{3} g=n_{1}+n_{2}$ with $c^{\prime 4 g / 3}$.

(ii-c) $\mathrm{p}=-\frac{1}{2}$ For $p=-\frac{1}{2}$, we have

$$
g\left(\sigma^{-\frac{1}{2}} y\right)=e^{c^{\prime} \sigma \frac{1}{y}}
$$

Using the same integral for $U\left(\sigma_{1}, \sigma_{2}\right)$ in terms of the $g(y)$, we find from the residues at $y_{1}=0$ and $y_{2}=0$

$$
U\left(\sigma_{1}, \sigma_{2}\right)=0
$$

In this case, we note that the selection rule is somewhat strange. Indeed the equality $3 g-$ $3+s=\sum n_{i}+(g-1)\left(1-\frac{2}{p}\right)+\sum l_{i}$, becomes for $p=-\frac{1}{2}, s=2$,

$$
-2 g+4=n_{1}+n_{2}-2\left(l_{1}+l_{2}\right)
$$

The $\sigma$ dependence is $\sigma^{(2 g-1)\left(1+\frac{1}{2}\right)}=\sigma^{-(2 g-1)}$. The expansion in powers of $\sigma$ assumed that the power $-(2 g-1)$ was positive which is not satisfied for positive $g$. This strange situation occurs only for $p=-\frac{1}{2}$. The vanishing result (94) for the two-point correlation function $U\left(\sigma_{1}, \sigma_{2}\right)$ may correspond to this strange selection rule. For the other cases, the two-point function is computed as in the case of the R-puncture for $p=\frac{1}{2}$, and the selection rule is satisfied. 


\section{Random Supermatrices and Duality}

We first briefly summarize the results of our previous study of random supermatrices, since it is needed for the present discussion. We refer for more details to [39].

A supermatrix $M$ is of the form

$$
M=\left(\begin{array}{cc}
a & \alpha \\
\bar{\alpha} & b
\end{array}\right)
$$

where $a$ and $b$ are $n \times n$ and $m \times m$ Hermitian matrices, respectively. The rectangular matrices $\alpha$ and $\bar{\alpha}$ are $n \times m$ and $m \times n$ respectively, and their elements are Grassmannian (i.e. anticommuting) variables.

The average of characteristic polynomials of the random supermatrix $M$, in the presence of an external source $A$, is defined by

$$
\begin{aligned}
F_{k}\left(\lambda_{1}, \ldots, \lambda_{k}\right) & =\frac{1}{Z_{N}}\left\langle\prod_{\alpha=1}^{k} \frac{1}{\operatorname{sdet}\left(\lambda_{\alpha} \cdot I-M\right)}\right\rangle_{A, M} \\
& =\frac{1}{Z_{N}} \int d M \prod_{\alpha=1}^{k} \frac{1}{\operatorname{sdet}\left(\lambda_{\alpha} \cdot I-M\right)} e^{\frac{i}{2} \operatorname{str} M^{2}+i \operatorname{str} M A}
\end{aligned}
$$

where $M$ and $A$ are supermatrices of the type (96), $I$ is the identity matrix and $Z_{N}$ is the normalization constant of the probability measure for $A=0$; the notations str and sdet stand for supertrace and superdeterminant [39]. We have to deal with a complex weight to make meaning of the integrals since

$$
s \operatorname{tr} \mathrm{M}^{2}=\operatorname{tr}\left(\mathrm{a}^{2}\right)-\operatorname{tr}\left(\mathrm{b}^{2}\right)+2 \operatorname{tr}()
$$

There is again a duality formula for (97) [39] which gives the same $F_{k}$ of (97) by another average

$$
F_{k}\left(\lambda_{1}, \ldots, \lambda_{k}\right)=e^{i \sum_{1}^{k} \lambda_{a}^{2} / 2} \int d B e^{\frac{i}{2} \operatorname{tr} B^{2}+i \sum_{a=1}^{k} \lambda_{a} B_{a a}} \frac{\prod_{j=1}^{m} \operatorname{det}\left(B-\rho_{j}\right)}{\prod_{i=1}^{n} \operatorname{det}\left(B-r_{i}\right)}
$$

where the external source $A$ is given by $A=\operatorname{diag}\left(r_{1}, \ldots, r_{n}, \rho_{1}, \ldots, \rho_{m}\right)$ and $B$ is a Hermitian $k \times k$ matrix.

This is again an $N-k$ duality, which exchanges the roles of the size $N=n+m$ of the matrices and of $k$ the number of points, between (97) and (99). The large $N$ scaling limit for the expectation values of the super-characteristic polynomials may then be approached from the dual representation (99) after tuning of the external source matrix $A$.

Note that if the "lower" eigenvalues $\rho_{j}=0(\mathrm{j}=1, \ldots, \mathrm{m})$ of the external source vanish, we obtain a simple determinant from (99)

$$
F_{k}\left(\lambda_{1}, \ldots, \lambda_{k}\right)=e^{i \sum_{1}^{k} \lambda_{a}^{2} / 2} \int d B(\operatorname{det} B)^{m} e^{\frac{i}{2} \operatorname{Tr} B^{2}+i \sum_{a=1}^{k} \lambda_{a} B_{a a}-\sum_{1}^{n} \operatorname{Tr} \log \left(B-r_{i}\right)}
$$

Following the same strategy we expand $\log \left(B-r_{i}\right)$ in powers of $1 / r_{i}$ :

$$
\sum_{i=1}^{n} \log \left(1-B / r_{i}\right)=-\sum_{i=1}^{n} \frac{1}{r_{i}} B-\sum_{i=1}^{n} \frac{1}{2 r_{i}^{2}} B^{2}-\sum_{i=1}^{n} \frac{1}{3 r_{i}^{3}} B^{3}-\cdots
$$


Restricting now to sources fulfilling the conditions

$$
\sum_{i=1}^{n} \frac{1}{r_{i}}=0, \text { quad } \sum_{i=1}^{n} \frac{1}{r_{i}^{2}}=i n, \quad \sum_{i=1}^{n} \frac{1}{r_{i}^{3}}=i n
$$

we obtain from (100) the Kontsevich-Penner [43] model:

$$
F_{k}\left(\lambda_{1}, \ldots, \lambda_{k}\right)=\frac{1}{Z_{k}} \int d B e^{i \frac{n}{3} \operatorname{Tr} B^{3}+m \operatorname{Tr} \log \mathrm{B}+i \operatorname{Tr} B \Lambda}
$$

where $B$ is a $k \times k$ Hermitian matrix, and $\Lambda=\operatorname{diag}\left(\lambda_{1}, \ldots, \lambda_{k}\right)$. Indeed after the rescaling $B \rightarrow B / n^{1 / 3}$ the powers $B^{l}$ with $l>3$ vanish in the large $n$ scaling limit. Thus we have obtained the Kontsevich-Penner model from the supermatrix duality of (97) and (99). The supermatrix formulation provides a natural derivation of the logarithmic term of the Penner model. Note that the Kontsevich-Penner model (103) had been obtained earlier from a twomatrix model (or equivalently to a time dependent matrix model) [6].

As discussed in a previous article [6], by adjusting the constraints (102), we may obtain a generalized Airy matrix model with a logarithmic potential

$$
F_{k}\left(\lambda_{1}, \ldots, \lambda_{k}\right)=\frac{1}{Z_{k}} \int d B e^{\frac{c}{p+1} \operatorname{Tr} B^{p+1}+m \operatorname{Tr} \log \mathrm{B}+i \operatorname{Tr} B \Lambda}
$$

where $c$ is a constant given by the constraints on the source matrix.

If now, instead of letting all the $\rho_{j}$ eigenvalues of the source vanish, we perform also an expansion of the numerator $\prod_{j=1}^{m} \operatorname{det}\left(B-\rho_{j}\right)$ in the integrand of (99), we obtain

$$
\begin{aligned}
& (\operatorname{det} B)^{m} \exp \left[\sum_{j=1}^{m} \operatorname{Tr} \log \left(1-\frac{\rho_{j}}{B}\right)\right] \\
& =(\operatorname{det} B)^{m} \exp \left(-\operatorname{Tr}\left[\frac{1}{B} \sum_{j=1}^{m} \rho_{j}+\frac{1}{2 B^{2}} \sum_{j=1}^{m} \rho_{j}^{2}+\frac{1}{3 B^{3}} \sum_{j=1}^{m} \rho_{j}^{3}+\cdots\right]\right)
\end{aligned}
$$

This yields a generalized Kontsevich-Penner model with positive and negative powers of $B$,

$$
F_{k}\left(\lambda_{1}, \ldots, \lambda_{k}\right)=\frac{1}{Z_{k}} \int d B \exp \left[\frac{c}{p+1} \operatorname{Tr} B^{p+1}+\sum_{l} c_{l} \operatorname{Tr} \frac{1}{B^{l}}+m \operatorname{Tr} \log B+i \operatorname{Tr} B \Lambda\right]
$$

where the scaling limit is here a large- $m$ limit, with $c$ a constant, and $c_{l}=\frac{1}{l} \sum \rho_{j}^{l}$.

We now consider the $s$-point function defined as

$$
U\left(t_{1}, \ldots, t_{s}\right)=\left\langle\operatorname{str} e^{i t_{1} M} \cdots \operatorname{str} e^{i t_{s} M}\right\rangle
$$

where $t_{i}=-i \sigma_{i}(i=1,2, \ldots, s)$ are real parameters, str is the supertrace, and $M$ the supermatrix defined in (97). This correlation function is a generating function for the intersection numbers when the external source is chosen at prescribed critical values.

This correlation function $U\left(t_{1}, \ldots, t_{s}\right)$ may be related to (97). Indeed replacing

$$
\prod_{\alpha=1}^{k} \frac{1}{\operatorname{sdet}\left(\lambda_{\alpha} \cdot I-M\right)}=e^{-s \operatorname{st} \sum_{\alpha} \log \left(\lambda_{\alpha} \cdot I-M\right)}
$$


and taking all the $\lambda_{\alpha}=\lambda$ equal, we have for one marked point,

$$
\begin{aligned}
U(\sigma) & =\lim _{k \rightarrow 0} \frac{1}{k} \int d \lambda e^{\sigma \lambda} \frac{\partial}{\partial \lambda}\left\langle e^{k \operatorname{str} \log (\lambda-M)}\right\rangle \\
& =\int d \lambda e^{\sigma \lambda}\left\langle\operatorname{str} \frac{1}{\lambda-M}\right\rangle=\int d \lambda e^{\sigma \lambda}\langle\operatorname{str} \delta(\lambda-M)\rangle \\
& =\left\langle\operatorname{str} e^{\sigma M}\right\rangle
\end{aligned}
$$

This one-point function is the Fourier transform of the resolvent. In [39] we have established explicit expressions for the correlation functions (107) as contour integrals. For the one point function ii reads

$$
U(\sigma)=\frac{1}{\sigma} \oint \frac{d u}{2 i \pi} e^{-i \sigma u} \prod_{i=1}^{n} \frac{u-r_{i}+\sigma / 2}{u-r_{i}-\sigma / 2} \prod_{j=1}^{m} \frac{u-\rho_{j}-\sigma / 2}{u-\rho_{j}+\sigma / 2}
$$

For $m=0$, it reduces to the ordinary non-supersymmetric expression with external source $r_{i}$. The integration in the $u$-plane encircles all the poles of the integrand (see [39]).

Similarly the connected part of the two point function $U_{c}\left(\sigma_{1}, \sigma_{2}\right)$ for the supersymmetric case is,

$$
\begin{aligned}
U_{c}\left(\sigma_{1}, \sigma_{2}\right)= & \oint \frac{d u_{1}}{2 i \pi} \frac{d u_{2}}{2 i \pi} e^{-i \sigma_{1} u_{1}-i \sigma_{2} u_{2}} \prod_{1}^{n} \frac{\left(u_{1}-r_{i}+\sigma_{1} / 2\right)\left(u_{2}-r_{i}+\sigma_{2} / 2\right)}{\left(u_{1}-r_{i}-\sigma_{1} / 2\right)\left(u_{2}-r_{i}-\sigma_{2} / 2\right)} \\
& \times \prod_{1}^{m} \frac{\left(u_{1}-\rho_{j}-\sigma_{1} / 2\right)\left(u_{2}-\rho_{i}-\sigma_{2} / 2\right)}{\left(u_{1}-\rho_{j}+\sigma_{1} / 2\right)\left(u_{2}-\rho_{j}+\sigma_{2} / 2\right)} \\
& \times \frac{1}{\left(u_{1}-u_{2}-\sigma_{1} / 2-\sigma_{2} / 2\right)\left(u_{1}-u_{2}+\sigma_{1} / 2+\sigma_{2} / 2\right)}
\end{aligned}
$$

It generalizes the non supersymmetric case by the inclusion of $r_{i}$ and $\rho_{j}$ in the external source which provides an additional freedom. To discuss the Airy matrix model with a logarithmic potential (Kontsevich-Penner model), we have chosen the simplest external sources $r_{i}=1$ and $\rho_{j}=0$.

An interesting application of the above formulae concerns the admixture of R and NSpunctures in the two-point function $U\left(\sigma_{1}, \sigma_{2}\right)$. One can mix the case of $p=2$ (Kontsevich model) with a $p=\frac{1}{2}$ spin-curve in (111). As seen earlier the $p=\frac{1}{2}$, is of R-type (fermionic vertex insertion) where as $p=2$ corresponds to an NS-puncture (bosonic vertex insertion).

The scattering amplitudes for superstring theory is related to the super Riemann surfaces with R and NS-punctures. Our present approach with super random matrices in an external source, provides the intersection numbers for punctures of both types at arbitrary genus. For two different spins $p$ and $p^{\prime}$, the $n$-point function, with a source choice similar to (4) for $r_{i}$ and $\rho_{j}$, reads

$$
\begin{aligned}
& U\left(\sigma_{1}, \ldots, \sigma_{n}\right)=\left\langle\operatorname{tr} e^{\sigma_{1} M} \cdots \operatorname{tr} e^{\sigma_{n} M}\right\rangle \\
& =\oint \prod_{i=1}^{n} \frac{d u_{i}}{2 i \pi} e^{c_{1} \sum_{i=1}^{n}\left[\left(u_{i}+\frac{\sigma_{i}}{2}\right)^{p+1}-\left(u_{i}-\frac{\sigma_{i}}{2}\right)^{p+1}\right]} e^{c_{2} \sum_{i=1}^{n}\left[\left(u_{i}+\frac{\sigma_{i}}{2}\right)^{p^{\prime}+1}-\left(u_{i}-\frac{\sigma_{i}}{2}\right)^{p^{\prime}+1}\right]} \\
& \quad \times \operatorname{det} \frac{1}{u_{i}-u_{j}+\frac{1}{2}\left(\sigma_{i}+\sigma_{j}\right)}
\end{aligned}
$$

with $c_{1}=\frac{n}{p^{2}-1} \sum_{i=1}^{p-1} \frac{1}{r_{i}^{p+1}}$, and $c_{2}=\frac{m}{p^{\prime 2}-1} \sum_{i=1}^{p^{\prime}-1} \frac{1}{\rho_{i}^{p^{\prime}+1}}$. 
The parameters $c_{1}$ and $c_{2}$ are associated with the spin $p$ and $p^{\prime}$, and they are useful for distinguishing the two different types, for instance for the case when $p$ is of NS- type and $p^{\prime}$ of R-type. An example is $p=2$ and $p^{\prime}=\frac{1}{2}$, since they belong to NS and R-types, respectively, as we have seen in Sect. 3.

We investigate the admixture of NS and R-punctures with $p=2$ and $p^{\prime}=\frac{1}{2}$.

$$
\begin{aligned}
U_{c}\left(\sigma_{1}, \sigma_{2}\right)= & \left\langle\operatorname{tr} e^{\sigma_{1} M} \operatorname{tr} e^{\sigma_{2} M}\right\rangle_{c} \\
= & -\oint \prod_{i=1}^{2} \frac{d u_{i}}{2 i \pi} e^{c_{1} \sum_{i=1}^{2}\left[\left(u_{i}+\frac{\sigma_{i}}{2}\right)^{p+1}-\left(u_{i}-\frac{\sigma_{i}}{2}\right)^{p+1}\right]} e^{c_{2} \sum_{i=1}^{2}\left[\left(u_{i}+\frac{\sigma_{i}}{2}\right)^{p^{\prime}+1}-\left(u_{i}-\frac{\sigma_{i}}{2}\right)^{p^{\prime}+1}\right]} \\
& \times \frac{1}{\left(u_{1}-u_{2}+\frac{1}{2}\left(\sigma_{i}+\sigma_{j}\right)\right)\left(u_{2}-u_{1}+\frac{1}{2}\left(\sigma_{i}+\sigma_{j}\right)\right)}
\end{aligned}
$$

The selection rule is,

$$
3 g-3+s=n_{1}+n_{2}+(g-1)\left(1-\frac{2}{\tilde{p}}\right)+\frac{1}{\tilde{p}}\left(l_{1}+l_{2}\right)
$$

in which $\tilde{p}$ means either $p$ or $p^{\prime}$ and here $s=2$.

For $p=2$, we take $l=0$ as NS-puncture, and for $p^{\prime}=\frac{1}{2}$, the puncture is of R-type with $l=-1$. We take $l_{1}=0$, and $l_{2}=-1$. For $p=2$, the factor $(g-1)\left(1-\frac{2}{p}\right)$ vanishes, while for $p^{\prime}=\frac{1}{2}$, it is equal to $-3(g-1)$. Therefore, the above selection rule reads

$$
6 g-6+s=n_{1}+n_{2}-2
$$

with $U_{c}\left(\sigma_{1}, \sigma_{2}\right) \sim \sum \sigma_{1}^{n_{1}+\frac{1}{2}} \sigma_{2}^{n_{2}}$, with punctures of NS-type $\left(\sigma_{1}\right)$ and $\mathrm{R}\left(\sigma_{2}\right)$, respectively.

We have discussed negative values of $p$ for the matrix model of Sect. 3. Matrix models with a logarithmic potential and negative powers of the matrices have been discussed in connection with superconformal gauge fields in the irregular conformal limit [40,41].

\section{Open Intersection Numbers}

As we have discussed in the previous section, an Airy matrix model with a logarithmic potential (Kontsevich-Penner model) may be derived from (103) and its intersection numbers are deduced from the generating functions $U\left(\sigma_{1}, \ldots, \sigma_{n}\right)$ by an appropriate tuning of the external matrix source.

For $p=2$ the one point function $U(\sigma)$ with a boundary reads

$$
\begin{aligned}
U(\sigma) & =\frac{1}{\sigma} \oint \frac{d u}{2 i \pi} e^{-\frac{c}{3}\left[\left(u+\frac{\sigma}{2}\right)^{3}-\left(u-\frac{\sigma}{2}\right)^{3}\right]+m \log \left(u+\frac{\sigma}{2}\right)-m \log \left(u-\frac{\sigma}{2}\right)} \\
& =\frac{1}{\sigma} e^{-\frac{c}{12} \sigma^{3}} \oint \frac{d u}{2 i \pi} e^{-c \sigma u^{2}+m \log \left(u+\frac{1}{2} \sigma\right)-m \log \left(u-\frac{1}{2} \sigma\right)}
\end{aligned}
$$

The constant $c$ is related to the normalization of the eigenvalues of the external source as in (106). Let us denote

$$
\sigma=\frac{1}{\lambda}, \quad t_{n}=\frac{1}{\lambda^{n+\frac{1}{2}}} .
$$


For $m=0$, we have

$$
U(\sigma)=\sqrt{\frac{\pi}{c}} \sum_{g=1}^{\infty} \frac{(-c)^{g}}{(12)^{g} g !} t_{3 g-2}
$$

From the above expression, we deduce the intersection number $\left\langle\tau_{3 g-2}\right\rangle$,

$$
\left\langle\tau_{n}\right\rangle=\frac{1}{(24)^{g} g !}
$$

where $n$ is given by Riemann-Roch formula (which will be discussed below in (128)), as $n=3 g-2$.

We note here the low orders for the intersection numbers for $m \neq 0$, computed from the integral (116),

$$
\begin{aligned}
\left\langle\tau_{1}\right\rangle & =\frac{1+12 m^{2}}{24}, \quad\left\langle\tau_{\frac{5}{2}}\right\rangle=\frac{m+m^{3}}{12}, \quad\left\langle\tau_{4}\right\rangle=\frac{1+56 m^{2}+16 m^{4}}{1152} \\
\left\langle\tau_{\frac{11}{2}}\right\rangle & =\frac{12 m+25 m^{3}+3 m^{5}}{2880}, \ldots
\end{aligned}
$$

These numbers have been obtained earlier from the Virasoro equations, within the replica method in [21], and the results of both methods agree.The above results coincide also with those of [45], if we replace $m$ by their parameter $N$, which is the size of the matrices. The indices $n$ of $\tau_{n}$ in (120) are integers or half-integers. If $n$ is a half-integer, the marked point is considered to be located on the boundaries.

The one point intersection numbers (120) are easily obtained from $U(\sigma)$ for $m \neq 0$. After rescaling of $u$, one has

$$
U(\sigma)=\frac{e^{-\frac{c}{12} \sigma^{3}}}{2 \sigma^{3 / 2}} \oint \frac{d u}{2 i \pi} e^{-\frac{c}{4} u^{2}+m \log \left(\frac{u+\sigma^{3 / 2}}{u-\sigma^{3 / 2}}\right)}
$$

The logarithmic term is expanded in powers of $\sigma^{3 / 2}$

$$
\log \left(\frac{u+\sigma^{3 / 2}}{u-\sigma^{3 / 2}}\right)=\frac{2}{u} \sigma^{3 / 2}+\frac{2}{3 u^{3}} \sigma^{9 / 2}+\frac{2}{5 u^{5}} \sigma^{15 / 2}+\cdots
$$

For odd powers of $u$ in the integrand of (121), i.e. $u^{-(2 j+1)}$, the integral is just a contour integral around $u=0$.

$$
\oint \frac{d u}{2 i \pi} \frac{e^{-a u^{2}}}{u^{2 k+1}}=\frac{(-a)^{k}}{k !}
$$

For even powers of $u$, i.e. $u^{-2 j}$ in the integrand, the integration becomes non-local. The following integral $I$ leads to $\Gamma$ - functions,

$$
\begin{aligned}
I & =\int_{-\infty}^{\infty} d u e^{-a u^{2}} \frac{1}{u^{2 k}}=\int_{0}^{\infty} d t t t^{-\frac{1}{2}-k} e^{-a t} \\
& =a^{k-\frac{1}{2}} \Gamma\left(\frac{1}{2}-k\right)=(-1)^{k} \frac{2^{k} \sqrt{\pi}}{(2 k-1) ! !} a^{k-\frac{1}{2}}
\end{aligned}
$$

in which a continuation from positive to negative $k$ has been used. 
For instance, for even powers of $m$, we have up to order $m^{2} \sigma^{3}$,

$$
\begin{aligned}
U(\sigma) & =e^{-\frac{c \sigma^{3}}{12}} \frac{1}{2 \sigma^{3 / 2}} \int e^{-\frac{c}{4} u^{2}}\left(1+2 m^{2} \sigma^{3} \frac{1}{u^{2}}\right) \\
& =\frac{1}{2 \pi} \sqrt{\frac{\pi}{c}} e^{-\frac{c \sigma^{3}}{12}} \frac{1}{\sigma^{3 / 2}}\left(1-c m^{2} \sigma^{3}\right)
\end{aligned}
$$

which indeed reproduces the result (120),

$$
\left\langle\tau_{1}\right\rangle=\frac{1}{24}\left(1+12 m^{2}\right)
$$

Using the formula of (123) and (124), we have derived the one-point intersection numbers to all orders in the genus, and they agree with the results derived by other methods $[21,45]$.

In the Appendix we recall how to use the Virasoro constraints for a matrix model with a logarithmic potential. This leads to explicit results for the intersection with NS and R punctures.

Number of boundary components for the Kontsevich-Penner model The parameter $m$ of the Kontsevich-Penner model, ( $m$ is the coefficient of the logarithmic potential), is related to the number of boundaries as follows. The number $b$ of the boundary components appears as the power of $m$, i.e. $m^{b}[45,46]$. To convince oneself of the validity of this interpretation one considers the expansion

$$
e^{m \operatorname{trl} \log M}=(\operatorname{det} M)^{m}=\sum_{b=0}^{\infty} \frac{1}{b !} m^{b}(\operatorname{trl} \log M)^{b}
$$

Thus the correlation function $U(s)$ has $b$-boundaries, described by the insertion of $\operatorname{tr} \log M=$ $\log \operatorname{det} M$. In general, we could consider the moduli space with genus $g, \mathrm{n} b$ boundaries, $n$ interior marked point, and $k$ marked points at the boundary. This moduli space is denoted as $M_{g, b ; k, n}$.

Since the Riemann surface with boundary becomes a Klein surface, we interpret it as a double surface $D \Sigma$ (D means double), where $\Sigma$ has genus $g$, with $b$ boundary components and $n$ interior marked points. The surface $D \Sigma$ has a doubled genus $g=2 h+b-1$, where $h$ is the number of handles and $b$ the number of boundary components. We have assumed here that there are no marked points at the boundary. From the dimensional constraint, the Riemann-Roch theorem gives

$$
3 g-3+s=6 h-6+3 b+2 n=2 \sum_{i=1}^{n} n_{i}
$$

where $n$ is the number of interior marked points (double counting; $\mathrm{s}=2 \mathrm{n}$ ) and $n_{i}$ the indices of the intersection numbers $\left\langle\prod_{i=1}^{n} \tau_{n_{i}}\right\rangle, b$ is the number of boundaries (holes) and $n$ is the number of punctures.

For the one point correlation, we have seen that when the intersection numbers are expressed as polynomials in $m$, when the power of $m$ is odd, the coefficient is obtained by a residue calculation, and it leads to an R-type. If the power of $m$ is even, then the puncture is of NS-type.

The interpretation of the Kontsevich-Penner model for the moduli space with boundaries does not refer to marked points at the boundaries. It has been argued that when $m=1$, it describes the moduli space with marked point at the boundaries [47]. In any case, it does not answer the question about how these marked points are distributed on the boundaries. To 
answer this question of the distribution of marked points, a refined open Kontsevich-Penner model has been proposed $[48,49]$.

\section{Summary}

In this article we have considered matrix models with a $p$-spin structure which generalize Kontsevich Airy matrix model. The external source plus duality method that we have used, provides explicit integral representations for the generating functions of the intersection numbers. Therefore these matrix models provide an alternative approach to the computation of the intersection numbers.

The integral representations of the generating functions present $p$ Stokes domains which characterize the so-called spin-structure in our formulation of the problem. The value $l=-1$ of the spin component corresponds to a Ramond puncture, but such punctures are not present for integer $p$. However our formulation allows for a continuation to non-integer and negative values of $p$. We could then show that the matrix models for half-integer spins $p$ do present R-type punctures. The results that we have found for the intersection numbers confirm the selection rule given by the Riemann-Roch relation, and allow for an extension of this rule to half-integer spins.

We have also used integrals over super-matrices that we had introduced in an earlier work, within the same framework of external source and explicit correlation functions. The scaling limit leads then to a natural extension to matrix models with logarithmic potentials which are known to generate surfaces with boundaries which are also considered in this work.

We intend to study future extensions to more general symmetries.

Acknowledgements We are thankful to Edward Witten for a correspondence about Ramond punctures. S.H. is partially supported by JSPS KAKENHI, 19H01813. E.B. thanks Okinawa Institute of Science and Technology (OIST) for its kind hospitality.

Open Access This article is licensed under a Creative Commons Attribution 4.0 International License, which permits use, sharing, adaptation, distribution and reproduction in any medium or format, as long as you give appropriate credit to the original author(s) and the source, provide a link to the Creative Commons licence, and indicate if changes were made. The images or other third party material in this article are included in the article's Creative Commons licence, unless indicated otherwise in a credit line to the material. If material is not included in the article's Creative Commons licence and your intended use is not permitted by statutory regulation or exceeds the permitted use, you will need to obtain permission directly from the copyright holder. To view a copy of this licence, visit http://creativecommons.org/licenses/by/4.0/.

\section{Appendix: Intersection Numbers for Logarithmic Potentials}

With the logarithmic potential introduced in [21], we have for $p=2$ (Kontsevich model),

$$
Z=\int d B e^{\operatorname{tr}\left(-\frac{1}{3} B^{3}+B \Lambda+m \log B\right)}
$$

where $B$ is an Hermitian matrix: it is an extension of (1) for $p=2$, and $F=\log Z$ has now additional terms $\Delta F$ for $m \neq 0$ as in [21],

$$
\Delta F=\frac{1}{2} m t_{0} t_{\frac{1}{2}}+\frac{1}{4} m^{2} t_{1}+\frac{1}{16} m^{2} t_{1}^{2}+\frac{m}{4} t_{0} t_{\frac{1}{2}} t_{1}+\frac{1}{24} m t_{\frac{1}{2}}^{3}+\frac{1}{4} m^{2} t_{\frac{1}{2}} t_{\frac{3}{2}}+\cdots
$$


Note that the $t_{\frac{1}{2}}$ can be written as $\left(t_{1,-1}\right)$ according to the previous notation of $t_{n, l}$, which is $\sum \frac{1}{\lambda_{i}^{n+\frac{1}{p}(l+1)}}$. It belongs to an R-puncture since it has $l=-1$. The Virasoro equations follow from the constraints

$$
\int d B \frac{\partial}{\partial B_{b a}} e^{\operatorname{tr}\left(-\frac{1}{3} B^{3}+B \Lambda+m \log B\right)}=0
$$

i.e.

$$
\left(-\left(\frac{\partial}{\partial \Lambda}\right)_{a b}^{3}+\left(\Lambda^{T} \frac{\partial}{\partial \Lambda}\right)_{a b}+(k+m) \delta_{a b}\right) Z=0
$$

The partition function $Z$ may then be obtained for finite $k$ ( $k$ is a size of the matrix $B$ ), as an expansion in inverse powers of the $\lambda_{i}[21]$.

There are no R-punctures in the Kontsevich model without logarithmic potential, but Rpairs do appear in the presence of a logarithmic potential, in the case of even powers of $m$, as seen in the last term of (130) $\frac{1}{4} m^{2} t_{\frac{1}{2}} t_{\frac{3}{2}}=\frac{1}{4} m^{2} t_{1,-1} t_{2,-1}$. The power of $m$ gives the number of boundaries. The existence of a Ramond sector for a logarithmic potential had already been noticed in [21].

When $p=2$ with logarithmic potential, the string equation is

$$
\left(-\frac{\partial}{\partial t_{0,0}}+\frac{1}{4} t_{0,0}^{2}-\frac{m}{2} t_{1,-1}+\sum_{n=0, \frac{1}{2}, 1, \frac{3}{2}, 2, \ldots}\left(n+\frac{1}{2}\right) t_{n+1,0} \frac{\partial}{\partial t_{n, 0}}\right) g=0
$$

where $Z=Z_{0} g$ with

$$
Z_{0}=\frac{1}{\prod_{i<j}\left(\sqrt{\lambda_{i}}+\sqrt{\lambda_{j}}\right)^{\frac{1}{2}}} e^{\frac{2}{3} \sum \lambda_{i}^{\frac{3}{2}}} \prod \lambda_{i}^{\frac{m}{2}}
$$

The string equation leads to

$$
\left\langle\tau_{0,0} \prod_{i} \tau_{n_{i}, l_{i}}\right\rangle=\sum_{i}\left\langle\tau_{n_{i}-1, l_{i}} \prod_{j \neq i} \tau_{n_{i}, l_{i}}\right\rangle
$$

In the presence of a logarithmic potential, there is an additional Virasoro equation for $t_{1,-1}$.

$$
\left(-2 \frac{\partial}{\partial t_{1,-1}}-m t_{0,0}-\left(\frac{1}{16}+\frac{m^{2}}{4}\right) t_{2,-1}-\frac{1}{12} J_{-4}^{(3)}+\frac{m}{4} J_{-4}^{(2)}-\frac{1}{2} J_{-1}^{(2)}\right) g=0
$$

where we have used the notations of $J_{-m}^{(l)}$ from [21]. The intersection numbers are tabulated in [45].

The string equation works also for the R-punctures. For instance, we have the relation,

$$
\left\langle\tau_{0,0} \tau_{4,-1}^{2}\right\rangle=2\left\langle\tau_{3,-1} \tau_{4,-1}\right\rangle=\frac{1}{144} m^{2}\left(m^{4}+11 m^{2}+16\right)
$$

The intersection numbers, with odd numbers of R-punctures, such as $\tau_{n,-1}$, may be expressed as polynomials in $m$, with odd powers of $m$. The intersection numbers, with an even number of R-punctures, are polynomials in $m$, with even powers of $m$. Hence, there is a parity for the numbers of R-punctures. 


\section{References}

1. 't Hooft, G.: A planar diagram theory for strong interactions, Nucl.Phys. B72, 461 (1974)

2. Stanford, D., Witten, E.: JT gravity and the ensembles of random matrix theory, arXiv:1907.03363

3. Brézin, E., Hikami, S.: Universal singularity at the closure of a gap in a random matrix theory. Phys. Rev, E 57, 4140 (1998)

4. Brézin, E., Hikami, S.: Intersection theory from duality and replica. Communication in Mathematical Physics 283, 507 (2008)

5. Brézin, E., Hikami, S.: Intersection numbers of Riemann surfaces from Gaussian matrix models. JHEP 10, 096 (2007)

6. Brézin, E., Hikami, S.: Computing topological invariants with one and two-matrix models. JHEP 04, 110 (2009)

7. Brézin, E., Hikami, S.: Random matrix, singularities and open/close intersection numbers. Journal of Physics A: Mathematical and Theoretical 48, 475201 (2015)

8. Brézin, E., Hikami, S.: Random matrix theory with an external source, SpringerBriefs in Mathematical Physics Vol. 19, Springer (2016)

9. Kontsevich, M.: Intersection theory on the moduli space of curves and the matrix Airy function. Commun. Math. Phys. 147, 1 (1992)

10. Mironov, A., Morozov, A., Semenov, G.W.: Unitary matrix integrals in the framework of generalized Kontsevich model 1. Brezin-Gross-Witten model, Intern. J. Mod. Phys. A, 11, 5031 (1996) arXiv:hep-th/9404005

11. Li, S., Troost, J.: Topological gravity with non-compact matter. JHEP 01, 158 (2019)

12. Witten, E.: Algebraic geometry associated with matrix models of two dimensions, in "Topological methods in modern mathematics", Publish or Perish, INC. P.235 (1993)

13. Witten, E.: The N matrix model and gauged WZW models. Nucl. Phys. B371, 191 (1992)

14. Harer, J., Zagier, D.: The Euler characteristic of the moduli space of curves. Invent. Math. 85, 457 (1986)

15. Penner, R.C.: Perturbative series and the moduli space of Riemann surfaces. J. Diff. Geometry 27, 35 (1988)

16. Witten, E., Gross, D.: Possible third order phase transition in the large N lattice gauge theory. Phys. Rev. D21, 446 (1980)

17. Brézin, E., Gross, D.: The external field problem in the large N limit of QCD. Phys. Lett. 97, 120 (1980)

18. Gross, D.J., Newman, M.J.: Unitary and Hermitian matrix models in an external field. 2: Kontsevich model and continuum Virasoro constraints. Nucl. Phys. B 380, 168 (1992)

19. Brézin, E., Hikami, S.: Duality and replicas for a unitary matrix model. JHEP 07, 067 (2010)

20. Norbury, P.: A new cohomology class on the moduli space of curves, arXiv:1712.03662

21. Brézin, E., Hikami, S.: On an Airy matrix model with a logarithmic potential. J. Phys. A: Mathematical and Theoretical 45, 045203 (2012)

22. Kac, V.G., Wakimoto, M.: Modular invariant representations of infinite-dimensional Lie algebras and superalgebras. Proc. Nat. Acad. Sci. USA 85, 4956 (1988)

23. Gaberdiel, M.R.: Fusion rules and logarithmic representation of a WZW model at fractional level. Nucl. Phys. B 618, 407 (2001)

24. Lesage, F., Mathieu, P., Rasmussen, J., Saleur, H.: The $\widehat{s u}(2)_{-\frac{1}{2}}$ WZW model and $\beta \gamma$ systems. Nucl. Phys. B647, 363 (2002). arXiv:hep-th/0207201

25. Nekrasov, N.A.: Lectures on curved beta-gamma systems, pure spinors, and anomalies, (2005) arXiv:hep-th/9511008

26. Li, K.: Topological gravity with minimal matter. Nucl. Phys. B354, 711 (1991)

27. Lewanski, D., Popolitov, A., Shadrin, S., Zvonkine, D.: Chiod formulas for the $r$-th roots and topological recursion, arXiv:1504.07439

28. Verlinde, E., Verlinde, H.: Solution of two-dimensional topological quantum gravity. Nucl. Phys. B348, 457 (1991)

29. Gomis, J., Komargodski, Z., Seiberg, N.: Phases of adjoint $\mathrm{QCD}_{3}$ and dualities. SciPost Phys. 5, 007 (2018)

30. Buryak, A., Clader, E., Tessler, R.J.: Closed extended r-spin theory and the Gelfand-Dickey wave function. Journal of geometry and physics 137, 132 (2019). arXiv:1710.04829

31. Buryak, A., Clader, E., Tessler, R.J.: Open r-spin theory II: The analogue of Witten's conjecture for r-spin disks, arXiv: 1809.02536

32. Witten, E.: The super period matrix with Ramond punctures. J. Geometry and Physics 92, 210 (2015). arXiv:1501.02499 
33. Witten, E.: Notes on super Riemann surfaces and their moduli. Pure and Applied Mathematics Quarterly 15, 57 (2019). arXiv:1209.2459

34. Jarvis, T., Kimura, T., Vaintrob, A.: Moduli spaces of higher spin curves and integrable hierarchies. Composite Math. 126, 157 (2001)

35. Mochizuki, T.: The virtual class of the moduli stack of stable $r$-spin curves. Commun. Math. Phys. 264, $1-40$ (2006)

36. Fan, H., Jarvis, T., Ruan, Y.: The Witten equation, mirror symmetry, and quantum singularity theory. Annals of Mathematics 178, 1-106 (2013)

37. Seeley, R., Singer, I.M.: Extending $\bar{\partial}$ to singular Riemann surfaces. JGP. 5, 121 (1981)

38. Rarita, W., Schwinger, J.: On a theory of particles with half-integral spin. Phys. Rev. 60, 61 (1941)

39. Brézin, E., Hikami, S.: Random supermatrices with an external source. JHEP 08, 086 (2018)

40. Gaiotto, D.: Asymptotically free $\mathrm{N}=2$ theories and irregular conformal block. J. Phys: Conf. Ser. 462, 012014 (2013). arXiv:0908.0307

41. Nishinaka, T., Rim, C.: Matrix models for irregular conformal blocks and Argyres-Douglas theories. JHEP 10, 138 (2012)

42. Brézin, E., Itzykson, C., Parisi, G., Zuber, J.B.: Planar diagrams. Commun. Math. Phys. 59, 35 (1978)

43. Brézin, E., Hikami, S.: Characteristic polynomials of random matrices. Commun. Math. Phys. 214, 111135 (2000)

44. Brézin, E., Hikami, S.: The intersection numbers of the $p$-spin curves from random matrix theory. JHEP 02, 035 (2013)

45. Bertola, M., Ruzza, G.: The Kontsevich-Penner matrix integral, isomonodromic tau functions and open intersection numbers. Ann. Henri Poincaré 20, 393-443 (2019). arXiv:1711.03360

46. Safnuk, B.: Combinatorial models for moduli spaces of open Riemann surfaces, arXiv:1609.07226

47. Alexandrov, A.: Open intersection numbers, Kontsevich-Penner model and cut-and-joint operators. arXiv: 1412.3772

48. Padharipande, R., Solomon, J. P., Tessler, R. J.: Intersection theory on moduli of disks, open KdV and Virasoro. arXiv:1409.2191

49. Alexandrov, A., Buryak, A., Tessler, R.: Refinded open intersection numbers and the Kontsevich-Penner matrix models. JHEP 03, 123 (2017). arXiv:1702.02319

Publisher's Note Springer Nature remains neutral with regard to jurisdictional claims in published maps and institutional affiliations. 